\title{
Low-energy spectrum of iron-sulfur clusters directly from many-particle quantum mechanics
}

\author{
Sandeep Sharma and Garnet Kin-Lic Chan* \\ Department of Chemistry, Frick Laboratory, Princeton University, NJ 08544 \\ Kantharuban Sivalingam and Frank Neese \\ Max Planck Institute for Chemical Energy Conversion, Muelheim, Germany
}

\begin{abstract}
FeS clusters are a universal biological motif. They carry out electron transfer, redox chemistry, and even oxygen sensing, in diverse processes including nitrogen fixation, respiration, and photosynthesis. The low-lying electronic states are key to their remarkable reactivity, but cannot be directly observed. Here we present the first ever quantum calculation of the electronic levels of [2Fe-2S] and [4Fe4S] clusters free from any model assumptions. Our results highlight limitations of long-standing models of their electronic structure. In particular, we demonstrate that the widely used HeisenbergDouble-Exchange model underestimates the number of states by 1-2 orders of magnitude, which can conclusively be traced to the absence of $\mathrm{Fe} \mathrm{d} \rightarrow \mathrm{d}$ excitations, thought to be important in these clusters. Further, the electronic energy levels of even the same spin are dense on the scale of vibrational fluctuations, and this provides a natural explanation for the ubiquity of these clusters in nature for catalyzing reactions.
\end{abstract}

Metals in enzymes perform remarkable chemistry under ambient pressures and temperatures. Among the most important cofactors are the iron-sulfur ( $\mathrm{FeS}$ ) clusters, comprising one to eight $\mathrm{Fe}$ atoms bridged by S ligands. In central processes of life ranging from nitrogen fixation to photosynthesis and respiration[1], these clusters perform diverse functions: redox chemistry, electron transfer, and even oxygen sensing. Their electronic structure, with multiple lowlying states with differing electronic and magnetic character, holds the key to this rich chemistry. However, uncovering the electronic structure has been highly non-trivial. Direct experimental assignment of these electronic levels in larger clusters has been impossible, since they lie at low energies and can be embedded within the vibrational modes of the clusters $[2,3]$.

Nonetheless, through intense collaboration in the last decades between experiment and theory, a consensus description of FeS cluster electronic states has emerged. This is based on the Heisenberg-Double-Exchange (HDE) model, combining Heisenberg exchange between Fe spins with a simplified version of Anderson's double-exchange $[4,5]$ for mixed valence delocalization. The HDE model was first proposed by Girerd[6] and Noodleman et al.[7, 8] for [2Fe-2S] dimers, and generalized to $[3 \mathrm{Fe}-4 \mathrm{~S}]$ and $[4 \mathrm{Fe}-4 \mathrm{~S}]$ clusters by Girerd, Münck and co-workers $[9,10]$ with further extensions in recent years[11, 12]. The HDE model has yielded many important insights. For example, the observed EPR $g<2$ in reduced [2Fe-2S] dimers was puzzling until it was recognized that the ground-state contains antiferromagnetic coupling of the $\mathrm{Fe}^{\mathrm{II}}$ and $\mathrm{Fe}^{\mathrm{III}}$ centers $[13,14]$. Similarly, in $[3 \mathrm{Fe}-4 \mathrm{~S}]$ and $[4 \mathrm{Fe}-4 \mathrm{~S}]$ clusters, HDE model mixed valence eigenstates provided the basis to interpret the distinctive Mössbauer, NMR, and ENDOR spectra[15-20].

Despite the many successes of this phenomenological model, its limitations are also well-known. The HDE model posits couplings a priori, which must be fitted before making predictions. In basic versions, couplings consist of Heisenberg exchange $J$ 's and double-exchange $B$ 's; extended versions include anisotropy, zero-field splitting, and further contributions[21]. An unambiguous determination of all parameters from experiment is clearly difficult, if not impossible. For example, in one famous case of a [4Fe-4S] cluster[1], the experimentally fitted $B$ 's range from $\approx 10 \mathrm{~cm}^{-1}$ to $\approx 600 \mathrm{~cm}^{-1}$, over two orders of magnitude! Further, FeS couplings obtained from broken-symmetry density functional theory (BS-DFT) computations (e.g. in pioneering work by Noodleman and others[8, 22-27]) are not clearly more reliable. This is because BS-DFT only describes a weighted average over the (multiplet) states, and individual parameters must once again be obtained through fitting; further they depend strongly on the density functional approximation used[28]. Beyond the above issues, a deeper criticism of the phenomenological models is that they make a priori choices about relevant chemical processes: a pure Heisenberg model will completely omit charge transfer phenomena. Without a reliable route to obtain model parameters, we cannot verify their validity, which raises the troubling question: how can we be sure that the current models are even qualitatively correct?

In principle, it should be possible to compute the states of the FeS clusters directly, without assuming an intermediate model. After all, the long-standing premise of ab-initio computation is that any molecule's electronic structure is obtainable, without assumptions, from the many-electron Schrödinger equation. Clearly, this has not yet been

*Electronic address: gkc1000@gmail.com 

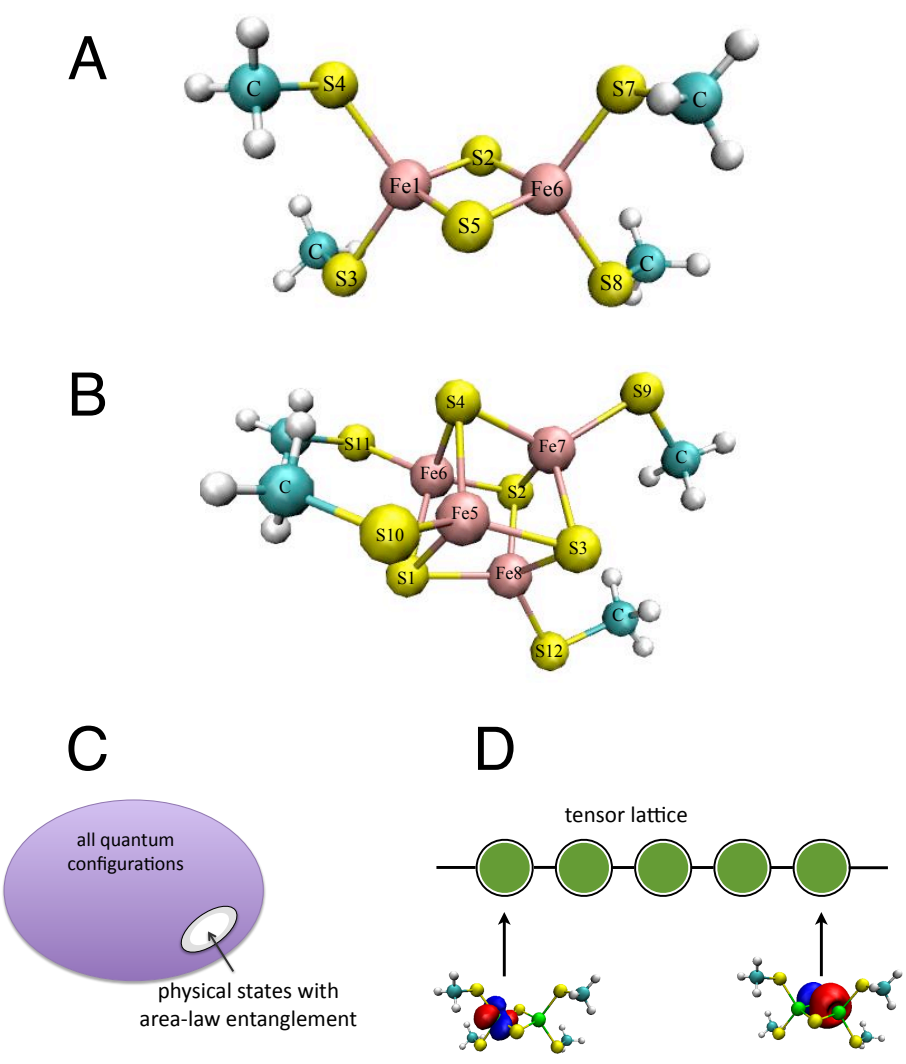

FIG. 1: Model clusters and many-electron wavefunctions used in this work. (A) [2Fe-2S] and (B) [4Fe-4S] clusters in this work, where the white circles denote $\mathrm{H}$ atoms. (Labels correspond to coordinates in Supplementary Tables 1, 2 and 11). (C) The area-law entanglement of the physical states can be used to reduce the complexity of quantum calculations. (D) Wavefunctions with area-law entanglement can compactly be written as a tensor network where each tensor (represented here by a circle) denotes an active space orbital and the bonds between adjacent orbitals introduce local entanglement between them. The network shown here is the linear network used by the density matrix renormalization group, which removes the need to model unphysical entanglement between widely separated sites on the chain. 
achieved for FeS clusters! Quantum mechanical computation is exponentially harder than classical simulation, because it supports superposition and non-local correlations known as entanglement. To avoid this complexity, practical simulations - for example those using DFT - rely on a mean-field approximation, which treats only classical-like quantum states without entanglement. This approximation completely fails in FeS clusters, however, due to the Fe d-shells, which are near-degenerate on the Coulomb interaction scale (so-called strongly interacting), rendering the molecular orbital picture and concept of a single mean-field electronic configuration invalid. This is why BS-DFT calculations, the standard computational method applied to FeS clusters, only provide an energetic average over the spectrum, and do not allow us to directly obtain the individual electronic states.

Strongly interacting electronic structure fundamentally involves entangled superpositions of many valence configurations. Malrieu and co-workers[29-32] showed that the main classes of configurations for metal centers include the configurations involving metal d electrons (a complete active space or CAS), augmented with excitations of bridging ligand orbitals, or by including ligand orbitals in the active space. However, even with these restrictions, the number of configurations grows exponentially with the number of atoms, and quickly becomes intractable. Early attempts to model these superpositions in the $[2 \mathrm{Fe}-2 \mathrm{~S}]$ dimer had to make further drastic approximations, completely removing non-bridging atoms, and including only d electrons in the active space [33]. In a [4Fe-4S] cluster, the minimal CAS comprises all Fe 3d and S 3p valence shells, a distribution of 54 electrons in 36 spatial orbitals, or $10^{16}$ configurations, unmanageable on any computer!

Recent advances from quantum information and condensed matter theory demonstrate, however, that physical quantum states - the quantum states seen in Nature - are special and contain a hidden structure to their wavefunctions. In particular, the low energy states possess area-law entanglement[34], reflecting the locality present in all physical systems regardless of interaction strength (Figure 1, panel C). This structure implies that the coefficients of the valence configurations in the FeS clusters are related in a special way, and the information compressed. To encode this relationship, we write the wavefunction as a tensor network[35], of which the density matrix renormalization group (DMRG) of White[36], a linear tensor network, is the most widely used example. A tensor in a tensor network represents a local variational degree of freedom: in a molecule, a tensor might represent an atom or an orbital; and contraction of the tensors creates the local entanglement, similar to a bond (Figure 1, panel D). The area-law implies that if we restrict ourselves to the physically relevant sector of quantum states the tensors used to describe physical states can be of low-rank. Working with low-rank tensor networks, we can elevate quantum simulations from the mean-field level to the level of the entangled quantum mechanics necessary to describe FeS clusters, while significantly ameliorating or in some cases completely bypassing the exponential complexity of the general quantum mechanical formulation.

Starting with the work of White and Martin[37], our group and others have been developing tensor networks and the DMRG in the context of quantum chemistry[38-46]. Here, we show that using our ab-initio DMRG methodology, we can now numerically solve the valence many-particle quantum mechanical equations for FeS clusters to chemical precision. This allows us, for the first time, to directly compute and probe the individual states and spectra in $\mathrm{FeS}$ clusters as large as the $[2 \mathrm{Fe}-2 \mathrm{~S}]$ and $[4 \mathrm{Fe}-4 \mathrm{~S}]$ clusters. This direct computation unshackles the discussion of $\mathrm{FeS}$ electronic structure from any earlier model assumptions. As we shall see, our calculations enable us to review, revisit and ultimately substantially revise, the historical models that have so far been the only basis for understanding electronic structure in these clusters, opening up the possibility to unlock the secrets of the chemistry of these clusters from direct simulation.

\section{RESULTS AND DISCUSSION}

\section{A. $[2 \mathrm{Fe}-2 \mathrm{~S}]$ dimers.}

We first begin with $[2 \mathrm{Fe}-2 \mathrm{~S}]$ dimers. We consider the synthetic $\left[\mathrm{Fe}_{2} \mathrm{~S}_{2}\left(\mathrm{SCH}_{3}\right)_{4}\right]^{2-/ 3-}$ complexes[47] that mimic the dimers prominently found in ferredoxins[48, 49] (Figure 1, panel A).

In canonical understanding based on the HDE model, the Fe atoms are placed in definite valence states. For the oxidized $[2 \mathrm{Fe}-2 \mathrm{~S}]^{2-}$ dimer, both Fe atoms are assumed high-spin $\mathrm{Fe}^{\mathrm{III}}\left(S_{1,2}=5 / 2\right)$, while for the reduced $[2 \mathrm{Fe}-2 \mathrm{~S}]^{3-}$ dimer, one is high-spin $\mathrm{Fe}^{\mathrm{II}}\left(S_{1}=2\right)$ and the other, high-spin $\mathrm{Fe}^{\mathrm{III}}\left(S_{2}=5 / 2\right)$. In the oxidized dimer there is assumed to be no double-exchange, and the HDE model reduces to the simpler Heisenberg form,

$$
H=2 J S_{1} \cdot S_{2}
$$

with levels $E(S)=J S(S+1)$ ( $S$ is total dimer spin, and $J$ is exchange coupling). In [2Fe-2S] dimers, $J>0$, thus the ground-state is low-spin with maximal antiferromagnetic alignment.

In the canonical picture of the reduced dimer, the additional electron can delocalize between the two Fe centers. (This is influenced by geometry and solvation, see e.g. Section 1.3 of the supplementary information, and Refs.[10, 50]). 


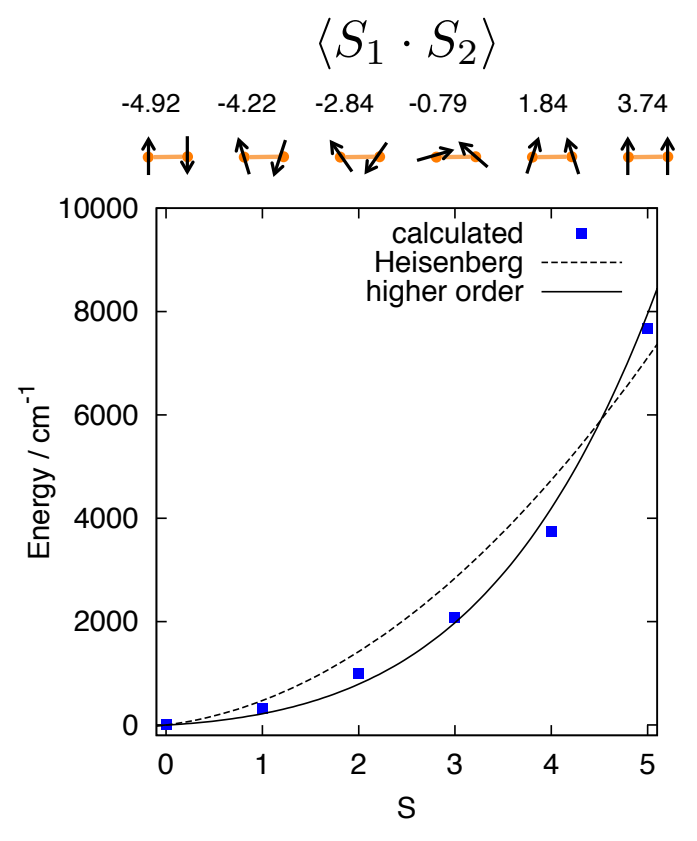

FIG. 2: Ab-initio levels of $\left[\mathrm{Fe}_{2} \mathrm{~S}_{2}\left(\mathrm{SCH}_{3}\right)_{4}\right]^{2-}$ and corresponding model fits. (Dashed line) Fit to Heisenberg model. (Solid line) Fit to Heisenberg plus quadratic couplings, capturing charge fluctuation effects. Top: Expectation value of the spin-correlation, $\left\langle S_{1} \cdot S_{2}\right\rangle$, for increasing dimer spin, showing progression from antiferromagnetic to ferromagnetic ordering.

In the HDE model[51, 52], this electron is placed in either a single bonding-type or antibonding-type orbital between the centers, splitting each Heisenberg level by a double-exchange contribution $B(S+1 / 2)$, and giving levels of the form

$$
E(S)=J S(S+1) \pm B(S+1 / 2)
$$

As discussed by Noodleman and Baerends[7], double-exchange stabilizes high-spin states.

We now examine the accuracy of these existing pictures by comparing against the electronic levels that can now be directly calculated using the ab-initio DMRG (details in Sections 1.1 and 1.2 of the supplementary information and methods section). Figure 2 shows the computed levels of the oxidized [2Fe-2S $]^{2-}$ complex as compared to the predictions of the Heisenberg model, Eq. (1). The exact DMRG levels qualitatively form a spin ladder from $S=0-5$, in general agreement with the Heisenberg model. As $S$ increases, the spin-spin correlation $\left\langle S_{1} \cdot S_{2}\right\rangle$ increases, transitioning from antiferromagnetic to ferromagnetic alignment. Fitting to Eq. (1) yields $J \approx 236 \mathrm{~cm}^{-1}$, which compares reasonably well to fits from magnetic susceptibility measurements on a similar synthetic dimer $(J \approx 148 \pm 16$ $\left.\mathrm{cm}^{-1}[51]\right)$ and computed BS-DFT estimates $\left(J \approx 310 \mathrm{~cm}^{-1}[52]\right)$. However, our ab-initio levels also show significant deviations from the level structure assumed by the traditional Heisenberg model. For example, the Heisenberg model overestimates the lower spin state energies, while underestimating those of the higher spin states. Measuring the local spin on the Fe atoms in our calculations we find $\left\langle S_{1}^{2}\right\rangle$ ranges from $5.47-5.74$ (for the $S=0, S=5$ states respectively), as compared with $\left\langle S_{1}^{2}\right\rangle=8.75$ for the pure $\mathrm{Fe}^{\mathrm{III}}(S=5 / 2)$ ion assumed in the model. This deviation from a pure $S=5 / 2$ ion illustrates the Heisenberg model's limits, which does not allow for additional spin or charge configuration mixing. In fact, charge fluctuations are responsible for the important spin delocalization onto the S orbitals, as previously observed by Noodleman[52]. Guihery and coworkers have derived the form of the corrections to the Heisenberg model that arise from quantum charge fluctuations[12]. In dimers, this yields a quadratic spin coupling $4 J_{Q}\left(S_{1} \cdot S_{2}\right)^{2} ;$ fitting to the ab-initio DMRG levels gives $J \approx 98 \mathrm{~cm}^{-1}$ and $J_{Q} \approx 6 \mathrm{~cm}^{-1}$. As demonstrated in Figure 2 , the quadratic coupling greatly improves the agreement, showing the importance of these corrections.

We now turn to the reduced $[2 \mathrm{Fe}-2 \mathrm{~S}]^{3-}$ dimer. Whereas the disagreement between the standard HDE model, and the directly computed ab-initio spectrum for the oxidized dimer, was primarily quantitative, for the reduced dimer the discrepancies are more severe. The HDE model predicts the splitting of the two lowest levels to increase with total spin, $2 B(S+1 / 2)$. However, in the ab-initio spectrum (Figure 3, panel A, red-curves) the splitting decreases with dimer spin from $S=1 / 2-3 / 2$. Clearly, this cannot be reproduced by any HDE model parameters, since the splitting is always proportional to $S$. (This remains after geometry relaxation, which introduces trapping (see Section 1.3 of the supplementary information)).

That the earlier model description breaks down for highly excited states is natural, but that it fails already for the 

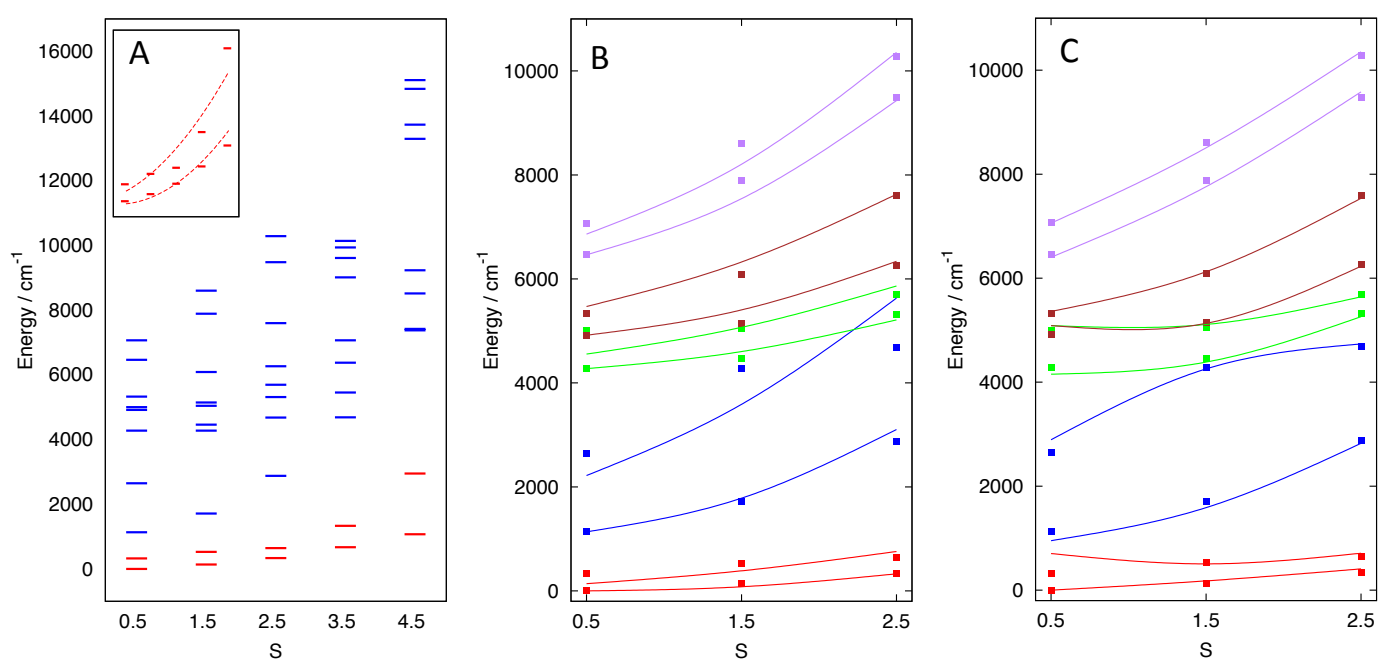

FIG. 3: Ab-initio levels of $\left[\mathrm{Fe}_{2} \mathrm{~S}_{2}\left(\mathrm{SCH}_{3}\right)_{4}\right]^{3-}$ and corresponding model fits. The multi-orbital Anderson model proposed here produces an excellent fit to all the levels, capturing the essential physics of the low energy states, while the single-pair and multi-pair HDE models do not. (A) Ab-initio levels of $\left[\mathrm{Fe}_{2} \mathrm{~S}_{2}\left(\mathrm{SCH}_{3}\right)_{4}\right]^{3-}$ for each dimer spin $S$. (Inset: Fit of lowest two levels (red) to the HDE model). The level separation does not increase monotonically as required in the HDE model, and the separation between the lowest two and higher levels is small (especially for $S=1 / 2,3 / 2,5 / 2$ ) indicating that the HDE model assumptions break down and multi-orbital double-exchange is important. (B) Fits $(S=1 / 2,3 / 2,5 / 2)$ to a multi-pair HDE model. Pairs of corresponding bonding and anti-bonding states are represented by the same color. (C) Fits $(S=1 / 2,3 / 2$, $5 / 2$ ) to a multi-orbital Anderson model. (B) and (C) use the same number of parameters, but (C) is much better than (B), demonstrating that multi-orbital double-exchange is not a sum of single exchange processes, as required by the multi-pair HDE model.
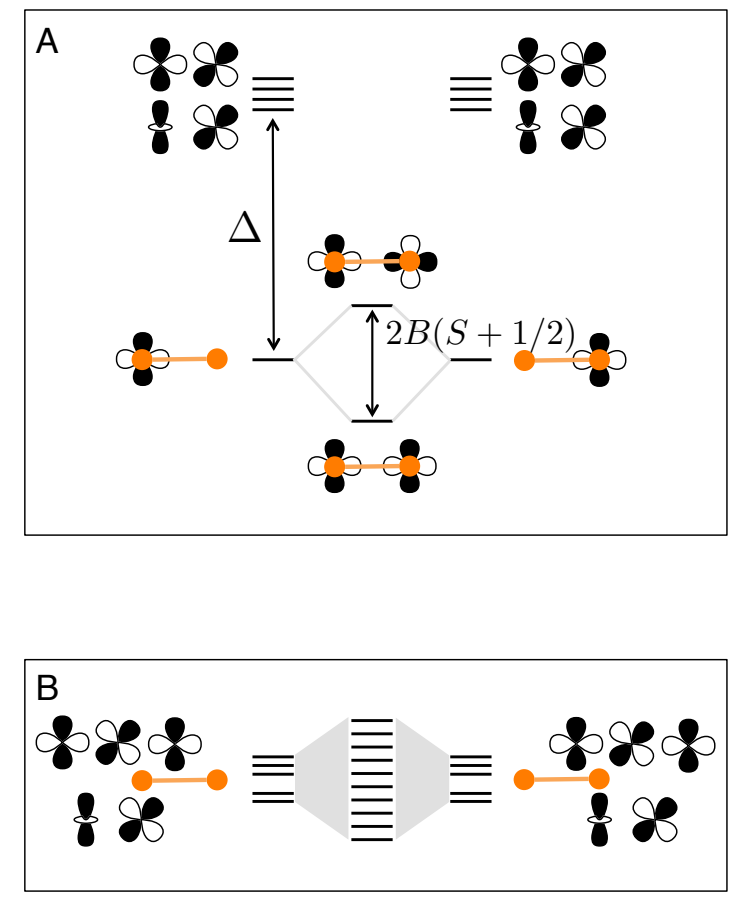

FIG. 4: Origin of the dense manifold in the reduced dimer. Although the HDE model predicts two low-lying states in the reduced dimer, the observed dense manifold can result from a ligand field splitting that is energetically comparable to the hopping energy. (A) The HDE model assumes a single electron hops between a pair of d orbitals on the Fe ions. This is valid if $\Delta$ is sufficiently large that other d orbitals are well separated. (B) In FeS systems, $\Delta$ is comparable to the hopping energy, giving a whole manifold of low-lying states at each dimer spin. 
lowest two states is surprising. As explained further below, this reflects the complicated nature of double-exchange in nearly orbitally degenerate metal ions. The HDE model assumes that the additional hopping electron is held in a single pair of prescribed bonding and antibonding orbitals bridging the the sulfur ligands. This is appropriate if ligand-field splitting places other bonding and antibonding orbitals at higher energies (panel A of Figure 4), but is not in fact the case for tetrahedrally coordinated $\mathrm{Fe}^{\mathrm{III}}$ ions where both orbitals of $e$-parentage (in $T_{d}$ symmetry) are near-degenerate, and weak ligand-field splitting by $\pi$-donor ligands leads to higher-lying orbitals of $t_{2}$-parentage also being accessible for double-exchange (panel B of Figure 4).

The multi-orbital nature of Fe double-exchange is immediately seen in the level spectrum at each dimer spin $S$ (Figure 3). Especially for $S=1 / 2,3 / 2,5 / 2$, the separation between the first two states and the higher states is small, rendering the single pair HDE model completely invalid. Instead, the lowest 10 levels constitute a dense manifold of related states, where the hopping electron occupies any one of 10 available d orbitals on the Fe atoms.

Multi-orbital double-exchange explains many of the earlier difficulties in determining consistent double-exchange parameters from experiment. Simply put, a well-defined global $B$ does not exist. Fitting the two lowest curves to the HDE model yields $J \approx 67 \mathrm{~cm}^{-1}$, and $B \approx 63 \mathrm{~cm}^{-1}$, while fitting the lowest and highest curves in the manifold, yields $J \approx 311 \mathrm{~cm}^{-1}$ and $B \approx 1052 \mathrm{~cm}^{-1}$. This wide range in $B$ values is consistent with earlier experimental fits, which also yield $B$ couplings varying by a factor of 50 or more [1]. (For a careful analysis of experimental variation in $B$, see Ref. [50]).

A naive way to extend the canonical HDE model to include multi-orbital exchange is to assume that the HDE energy levels in Eq. (2) simply generalize from 1 to 5 hopping pairs and 5 sets of $J_{i}$ and $B_{i}, i=1 \ldots 5$ couplings, with ligand-field splittings $\Delta_{i}, i=1 \ldots 4$. (Multi-pair HDE models have been considered for [4Fe-4S] clusters in the context of $\sigma$ versus $\delta$ delocalization pathways [22, 52]). This gives levels of the form

$$
E_{i}(S)=\Delta_{i}+2 J_{i} S_{1} \cdot S_{2} \pm B_{i}\left(S_{i}+1 / 2\right)
$$

where $i$ labels the orbital pair associated with the hopping. Fits to this multi-pair form are shown in Figure 3 (panel B). The multi-pair HDE model does capture the low-lying spectrum better, since it has more parameters, but it still cannot reproduce the non-monotonic behaviour in pairs of energy levels, such as the decreasing gap between the first two levels. This shows that multi-orbital exchange cannot be considered a simple pairwise process.

The correct qualitative picture for multi-orbital exchange requires a return to Anderson's original Hamiltonian for double-exchange. For this system it takes the form (detailed derivation in Section 1.4 of the supplementary information)

$$
H=\sum_{i} J_{i} s_{1 i} \cdot s_{2 i}+\sum_{i \sigma}\left[\beta_{i}\left(c_{1 i \sigma}^{\dagger} c_{2 i \sigma}+c_{2 i \sigma}^{\dagger} c_{1 i \sigma}\right)+\Delta_{i}\left(c_{1 i \sigma}^{\dagger} c_{1 i \sigma}+c_{2 i \sigma}^{\dagger} c_{2 i \sigma}\right)\right]
$$

( $i$ labels $d$ orbitals, $s_{1 i}, s_{2 i}$ are electron spins, and $c_{1 i}^{(\dagger)}, c_{2 i}^{(\dagger)}$ create and destroy electrons). Note that this Anderson Hamiltonian has the same number of parameters as a multi-pair HDE model (Eq. 3), but the fit (Figure 3, panel C) is much improved and now obtains the correct non-monotonic features. Further, the fit is stable, near unique, and yields reasonable parameters: ligand field splittings are approximately $4000-5000 \mathrm{~cm}^{-1}$, consistent with spectroscopic estimates for tetrahedral Fe (see Supplementary Table 10). Thus all qualitative features of our ab-initio spectrum can be understood by treating multi-orbital exchange in this more complete way.

In summary, our directly computed levels show that the low-lying spectrum of [2Fe-2S] dimers is much richer and denser than the simple pair-splitting long assumed within the canonical HDE picture; the level spectrum is generated by a complex multi-orbital exchange process, and this process cannot be viewed as a simple "sum" over single orbital double-exchange pathways.

\section{B. $[4 \mathrm{Fe}-4 \mathrm{~S}]$ clusters.}

We now turn from FeS dimers to the more complicated [4Fe-4S] clusters. As a representative of Nature's cubanes we consider the $\left.\left[\mathrm{Fe}_{4} \mathrm{~S}_{4}\left(\mathrm{SCH}_{3}\right)_{4}\right)\right]^{2-}$ cluster, derived from the synthetic cluster studied by Holm and coworkers [53]. The deduction of the cubane ground-state from experimental measurements is an early triumph of inorganic spectroscopy [19]. It is conventionally believed that the ground-state consists of two coupled iron dimers, located on opposite faces of the cube. The dimers are thought of as mixed-valence $\mathrm{Fe}^{2.5+}, \mathrm{Fe}^{2.5+}$ pairs coupled to a high-spin $S=9 / 2$ state, which further recouple in the ground-state to form an overall singlet.

Since we can compute the electronic structure of the $[4 \mathrm{Fe}-4 \mathrm{~S}]$ cluster directly (see Methods) we can now test the long-standing hypothesis for the ground-state. In our computed singlet ground-state, the spin density is zero everywhere (since our wavefunction is a true spin singlet), but the nature of the couplings can be established from spin correlation functions $\left\langle S_{i} \cdot S_{j}\right\rangle$ (Figure 5). These indicate ferromagnetic coupling along the top and bottom faces 

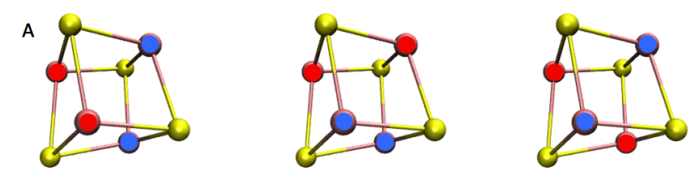

B
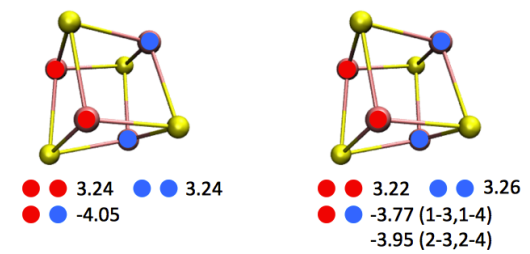

FIG. 5: Spin pairings in the $[4 \mathrm{Fe}-4 \mathrm{~S}]$ cluster. Unlike in a perfectly cubic cluster, where 3 equivalent pairings of Fe's exist, the geometry-optimized cluster breaks this symmetry and favors one pairing over the other two. The dimer pairs, and their relative spin orientations, can be detected through the spin correlation functions. (A) 3 equivalent pairings of Fe's into dimers in a perfect $[4 \mathrm{Fe}-4 \mathrm{~S}]$ cubane. Red-red: Ferromagnetic coupling, red-blue: Antiferromagnetic coupling. (B) Spin correlation functions $\left(\left\langle S_{i} \cdot S_{j}\right\rangle\right)$ for lowest singlet (left) and triplet (right) states of the $\left[\mathrm{Fe}_{4} \mathrm{~S}_{4}\left(\mathrm{SCH}_{3}\right)_{4}\right]^{2-}$ cluster.

with antiferromagnetic coupling between the faces, corresponding precisely to the experimental picture of two highspin $\mathrm{Fe}^{2.5+}, \mathrm{Fe}^{2.5+}$ dimers recoupled into a singlet, thus confirming the long-standing interpretation. We can further extend our calculations to the lowest triplet state: this lies $351 \mathrm{~cm}^{-1}$ above the singlet state. From its spin correlation functions, we see that the triplet corresponds to a spin-canted state: the $S=9 / 2$ dimer spins are tilted relative to their ground-state orientation.

What about the complete [4Fe-4S] spectrum? From the complexity of the $[2 \mathrm{Fe}-2 \mathrm{~S}]$ spectrum, we expect this will be a formidable beast. While the complete spectrum is too expensive, we can calculate some of the lower-lying states. The 10 lowest singlet $S=0$ and high-spin $S=9$ states are shown in Figure 6, as well as the lowest triplet, and a (more qualitative) spectrum for the 150 lowest $S=9$ states. In a perfect cubane we expect three degenerate singlet groundstates, from the three possible spin couplings in Figure 5. However, vibronic coupling distorts the ground-state of the $[4 \mathrm{Fe}-4 \mathrm{~S}]$ cluster, opening a gap between the ground- and higher-lying states. Neverthless, even in the distorted [4Fe- $4 \mathrm{~S}]$ cluster, the manifold of low-lying states remains accessible and dense on the $10-20 \mathrm{kcal} / \mathrm{mol}$ scale of biological FeS reorganization energies [54]. A detailed analysis of the [4Fe-4S] excited states and their coupling to distortions is presented in Section 2.4 of the supplementary information. Further, the structure of the low-lying spectrum, for any cluster spin $S$, is very different to the isolated 4 low-lying levels predicted by canonical HDE models for each $S$. This is expected as the model fails already in the $[2 \mathrm{Fe}-2 \mathrm{~S}]$ dimers, due to neglect of multi-orbital double exchange discussed above. Appropriate model Hamiltonians for the [4Fe-4S] clusters are analysed in detail in Section 2.4 of the supplementary information.

The high density of states (and their sensitivity to geometry via the vibronic coupling) provides an intriguing hypothesis behind the unusual chemical flexibility and ubiquity of the [4Fe- $4 \mathrm{~S}]$ clusters. Conventionally, molecular reactivity proceeds via well-defined potential energy surfaces. However, in [4Fe-4S] clusters, a large number of states of the same (and different) spin are energetically accessible during reorganization dynamics. This suggests that [4Fe$4 \mathrm{~S}]$ clusters can non-adiabatically switch between many different frontier electronic states in a reaction, in essence a generalization of two-state reactivity already postulated in single-Fe porphyrins[55] but without slow spin-forbidden state crossings. Coupling between the spectrum and geometry allows for "fine-tuning" of reactivity by the environment. $[4 \mathrm{Fe}-4 \mathrm{~S}]$ clusters thus appear to provide a conceptual bridge between molecular and surface catalytic reactivity. In the latter case, non-adiabatic processes on many potential energy surface are common, and greatly modify the timescales of electron and energy transfer[56]. 


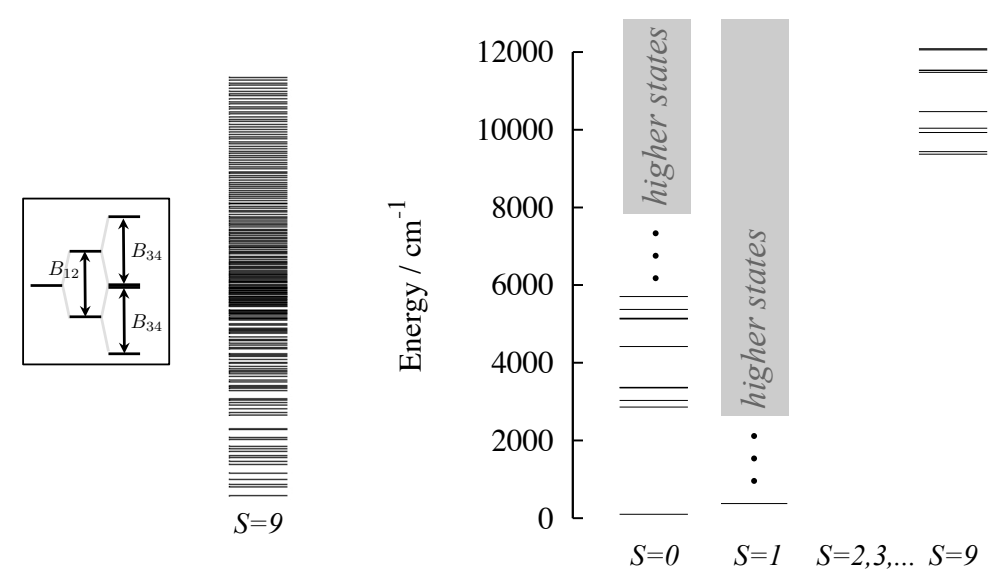

A

B

FIG. 6: Energy levels of the [4Fe-4S] cluster. The low-lying spectrum of the $[4 \mathrm{Fe}-4 \mathrm{~S}]$ cluster is unusually dense and is starkly different from the predictions of the simple HDE model. (A) Isolated 4 low-lying states predicted (for each $S$ ) from the HDE model, compared to the qualitative ab-initio density of states (shown for $S=9$ ), (B) Lowest lying 10 singlet $(S=0)$ and high spin $(S=9)$ states), as well as ground-state triplet $S=1$ state, showing the small energy scales and detailed structure of the spectrum.

\section{CONCLUSIONS}

To summarize, using simplifying entanglement structure of physical many-particle quantum states, we have computed the individual ground and excited state energy levels of [2Fe-2S] and [4Fe-4S] clusters without model assumptions. Direct access to these energy levels has not previously been possible either through experiment or theory. Our calculations have allowed us to critically examine the validity of the consensus phenomenological models that have so far been the only way to understand FeS chemistry. In both the [2Fe-2S] dimer and the [4Fe- $4 \mathrm{~S}]$ cluster, we find that earlier understanding based on the canonical Heisenberg-Double-Exchange model underestimates the number of low-lying states by 1-2 orders of magnitude. These low-lying states arise from multi-orbital double-exchange processes. The new level spectrum we reveal has important implications for reactivity, as the density and accessibility of the low-lying states argues for the importance of multiple electronic states and non-adiabaticity in reactions. The theoretical techniques described here are potentially applicable to biological systems of even greater complexity, including the M- and P-clusters of nitrogenase [57, 58]. More broadly, our work demonstrates new possibilities of realising spectroscopy in complex systems, by directly computing entangled electronic structure from many-particle quantum mechanics, without the need for a priori model assumptions. 


\section{METHODS}

All our density matrix renormalization group (DMRG) calculations employed the BLOCK code. Density functional calculations to obtain the initial geometries were carried out using the ORCA package. For DMRG calculations on the $[2 \mathrm{Fe}-2 \mathrm{~S}]$ clusters, we employed a full valence complete active space (all $\mathrm{Fe} 3 \mathrm{~d}$ and $\mathrm{S} \mathrm{3p}$ orbitals), and further include the $\mathrm{Fe} 4 \mathrm{~s}$ and $4 \mathrm{~d}$ shells to account for additional dynamic correlation effects. In the oxidized cluster, this corresponds to a 30 electron, 32 orbital active space. Even though the formal Hilbert space dimension is greater than $10^{17}$, our calculations are enabled by the presence of some special entanglement structure in the states. In the $[2 \mathrm{Fe}-2 \mathrm{~S}]$ clusters, we estimate that the electronic relative energies are converged to better than $0.1 \mathrm{kcal} / \mathrm{mol}(\approx 35$ $\mathrm{cm}^{-1}$ ) of the exact active space results. For the $[4 \mathrm{Fe}-4 \mathrm{~S}]$ clusters we employed an active space with all $\mathrm{Fe} 3 \mathrm{~d}$ and $\mathrm{S}$ $3 \mathrm{p}$ orbitals. These calculations were more expensive than the dimer calculations, thus the DMRG energy differences between the singlet and triplet states are converged to only about $0.5-1 \mathrm{kcal} / \mathrm{mol}$. These estimated errors refer to the errors from the corresponding complete active space result. Dynamical correlation may lead to further small changes, as discussed in the Section 1.2 of supplementary information. The higher singlet and high-spin states in Figure 6 were calculated to lower accuracy than the ground singlet and triplet states, but are qualitatively correct. The multiplet states are computed explicitly without assuming any mutual inter-relationship, in contrast to BS-DFT techniques, which only obtain the high-spin state and a weighted average of the multiplet energies, relying on model assumptions to deduce the individual levels [28]. All our computed wavefunctions exactly preserve spin $\left(S^{2}\right)$ symmetry, due to the use of an SU(2) invariant DMRG code developed in our lab[40]. A full discussion of all the active spaces, geometry, and examination of the DMRG convergence, is presented in the Sections 1.1, 1.2, 2.1 and 2.2 of the supplementary information. For the spin correlation functions, $S_{1}$ and $S_{2}$ are defined from local Fe spin operators, and $\left\langle S_{1} \cdot S_{2}\right\rangle$ is the ab-initio expectation value. These operators are defined in Equations 1 and 2 of the supplementary information.

\section{ACKNOWLEDGEMENTS}

Work performed by S.S. and G.K.C. was supported by the US National Science Foundation CHE-1265277, using software developed with the support of OCI-1265278. F.N. and K.S. gratefully acknowledge financial support of this work by the Max Planck Society, the University of Bonn and the SFB 813 "Chemistry at Spin Centers".

\section{AUTHOR CONTRIBUTIONS}

S.S. performed the DMRG calculations, analyzed the results and contributed to the writing of the manuscript. F.N. contributed to the writing of the manuscript. K.S. performed the geometry optimization for the [4Fe-4S] model cluster. G.K.C. wrote the manuscript and contributed to the calculations and analysis of the results. All authors discussed the results and commented on the manuscript.

[1] Beinert, H., Holm, R. H. \& Münck, E. Iron-sulfur clusters: nature's modular, multipurpose structures. Science 277, 653-659 (1997).

[2] Johnson, D. C., Dean, D. R., Smith, A. D. \& Johnson, M. K. Structure, function, and formation of biological iron-sulfur clusters. Annual Review of Biochemistry 74, 247-281 (2005).

[3] Noodleman, L., Peng, C. Y., Case, D. A. \& Mouesca, J. M. Orbital interactions, electron delocalization and spin coupling in iron-sulfur clusters. Coordination Chemistry Reviews 144, 199-244 (1995).

[4] Zener, C. Interaction between the d-shells in the transition metals. II. Ferromagnetic compounds of manganese with perovskite structure. Phys. Rev. 82, 403-405 (1951).

[5] Anderson, P. W. \& Hasegawa, H. Considerations on double exchange. Physical Review 100, 675-681 (1955).

[6] Girerd, J.-J. Electron transfer between magnetic ions in mixed valence binuclear systems. The Journal of Chemical Physics 79, 1766-1775 (1983).

[7] Noodleman, L. \& Baerends, E. J. Electronic structure, magnetic properties, ESR, and optical spectra for 2-iron ferredoxin models by LCAO-X $\alpha$ valence bond theory. Journal of the American Chemical Society 106, 2316-2327 (1984).

[8] Noodleman, L. \& Davidson, E. R. Ligand spin polarization and antiferromagnetic coupling in transition metal dimers. Chemical physics 109, 131-143 (1986).

[9] Papaefthymiou, V., Girerd, J. J., Moura, I., Moura, J. J. G. \& Muenck, E. Moessbauer study of D. gigas ferredoxin II and spin-coupling model for $\mathrm{Fe}_{3} \mathrm{~S}_{4}$ cluster with valence delocalization. Journal of the American Chemical Society 109, 4703-4710 (1987). 
[10] Blondin, G. \& Girerd, J. J. Interplay of electron exchange and electron transfer in metal polynuclear complexes in proteins or chemical models. Chemical Reviews 90, 1359-1376 (1990).

[11] Borshch, S. A., Kotov, I. N. \& Bersuker, I. B. A vibronic model for exchange-coupled mixed-valence dimers. Chemical Physics Letters 111, 264-270 (1984).

[12] Labeguerie, P. et al. Is it possible to determine rigorous magnetic Hamiltonians in spin s $=1$ systems from density functional theory calculations? The Journal of Chemical Physics 129, 154110 (2008).

[13] Gibson, J. F., Hall, D. O., Thornley, J. H. \& Whatley, F. R. The iron complex in spinach ferredoxin. Proceedings of the National Academy of Sciences 56, 987-990 (1966).

[14] Brintzinger, H., Palmer, G. \& Sands, R. H. On the ligand field of iron in ferredoxin from spinach chloroplasts and related nonheme iron enzymes. Proceedings of the National Academy of Sciences 55, 397-404 (1966).

[15] Rius, G. \& Lamotte, B. Single-crystal ENDOR study of an ${ }^{57}$ Fe-enriched iron-sulfur $\left[\mathrm{Fe}_{4} \mathrm{~S}_{4}\right]^{3+}$ cluster. Journal of the American Chemical Society 111, 2464-2469 (1989).

[16] Mouesca, J. M., Lamotte, B. \& Rius, G. Comparison between spin population distributions in two different $\left[\mathrm{Fe}_{4} \mathrm{~S}_{4}\right]^{3+}$ clusters by proton ENDOR in single crystals of a synthetic model compound. Journal of Inorganic Biochemistry 43, 251 (1991).

[17] Bertini, I., Briganti, F., Luchinat, C., Scozzafava, A. \& Sola, M. Proton NMR spectroscopy and the electronic structure of the high potential iron-sulfur protein from Chromatium vinosum. Journal of the American Chemical Society 113, 1237-1245 (1991).

[18] Banci, L. et al. Proton NMR spectra of oxidized high-potential iron-sulfur protein (HiPIP) from Rhodocyclus gelatinosus. A model for oxidized HiPIPs. Inorganic Chemistry 30, 4517-4524 (1991).

[19] Papaefthymiou, V., Millar, M. M. \& Muenck, E. Moessbauer and EPR studies of a synthetic analog for the iron-sulfur $\mathrm{Fe}_{4} \mathrm{~S}_{4}$ core of oxidized and reduced high-potential iron proteins. Inorganic Chemistry 25, 3010-3014 (1986).

[20] Kappl, R., Ebelshäuser, M., Hannemann, F., Bernhardt, R. \& Hüttermann, J. Probing electronic and structural properties of the reduced $[2 \mathrm{Fe}-2 \mathrm{~S}]$ cluster by orientation-selective ${ }^{1} \mathrm{H}$ ENDOR spectroscopy: Adrenodoxin versus Rieske iron-sulfur protein. Applied Magnetic Resonance 30, 427-459 (2006).

[21] Maurice, R., Guihery, N., Bastardis, R. \& de Graaf, C. Rigorous extraction of the anisotropic multispin hamiltonian in bimetallic complexes from the exact electronic Hamiltonian. Journal of Chemical Theory and Computation 6, 55-65 (2009).

[22] Noodleman, L., Norman, J. G., Osborne, J. H., Aizman, A. \& Case, D. A. Models for ferredoxins: electronic structures of iron-sulfur clusters with one, two, and four iron atoms. Journal of the American Chemical Society 107, 3418-3426 (1985).

[23] Mouesca, J.-M., Chen, J. L., Noodleman, L., Bashford, D. \& Case, D. A. Density functional/poisson-boltzmann calculations of redox potentials for iron-sulfur clusters. Journal of the American Chemical Society 116, 11898-11914 (1994).

[24] Shoji, M. et al. Theory of chemical bonds in metalloenzymes III: Full geometry optimization and vibration analysis of ferredoxin-type [2Fe-2S] cluster. International Journal of Quantum Chemistry 107, 116-133 (2007).

[25] Noodleman, L. Valence bond description of antiferromagnetic coupling in transition metal dimers. The Journal of Chemical Physics 74, 5737-5743 (1981).

[26] Yamaguchi, K., Fueno, T., Ueyama, N., Akira, N. \& Masaaki, O. Antiferromagnetic spin couplings between iron ions in ironsulfur clusters. a localized picture by the spin vector model. Chemical Physics Letters 164, 210 - 216 (1989).

[27] Yamaguchi, K., Fueno, T., Ozaki, M., Ueyama, N. \& Nakamura, A. A general spin-orbital (gso) description of antiferromagnetic spin couplings between four irons in iron-sulfur clusters. Chemical Physics Letters 168, 56 - 62 (1990).

[28] Neese, F. Prediction of molecular properties and molecular spectroscopy with density functional theory: From fundamental theory to exchange-coupling. Coordination Chemistry Reviews 253, 526-563 (2009).

[29] Miralles, J., Daudey, J.-P. \& Caballol, R. Variational calculation of small energy differences. The singlet-triplet gap in $\left[\mathrm{Cu}_{2} \mathrm{Cl}_{6}\right]_{2}$. Chemical Physics Letters 198, 555-562 (1992).

[30] Miralles, J., Castell, O., Caballol, R. \& Malrieu, J.-P. Specific CI calculation of energy differences: Transition energies and bond energies. Chemical Physics 172, 33-43 (1993).

[31] Castell, O. \& Caballol, R. Ab-initio configuration interaction calculation of the exchange coupling constant in hydroxo doubly bridged Cr(III) dimers. Inorganic Chemistry 38, 668-673 (1999).

[32] Cabrero, J., Ben Amor, N., de Graaf, C., Illas, F. \& Caballol, R. Ab-initio study of the exchange coupling in oxalato-bridged $\mathrm{Cu}(\mathrm{II})$ dinuclear complexes. The Journal of Physical Chemistry A 104, 9983-9989 (2000).

[33] Hübner, O. \& Sauer, J. The electronic states of fe2s $2^{-/ 0 /+/ 2+}$. The Journal of Chemical Physics 116, 617-628 (2002).

[34] Hastings, M. B. Entropy and entanglement in quantum ground states. Phys. Rev. B 76, 35114 (2007).

[35] Verstraete, F., Murg, V. \& Cirac, J. I. Matrix product states, projected entangled pair states, and variational renormalization group methods for quantum spin systems. Adv. Phys. 57, 143 (2008).

[36] White, S. R. Density matrix formulation for quantum renormalization groups. Phys. Rev. Lett. 69, 2863 (1992).

[37] White, S. R. \& Martin, R. L. Ab initio quantum chemistry using the density matrix renormalization group. J. Chem. Phys. 110, 4127 (1999).

[38] Chan, G. K.-L. \& Sharma, S. The Density Matrix Renormalization Group in Quantum Chemistry. Ann. Rev. Phys. Chem. 62, 465 (2011).

[39] Kurashige, Y., Chan, G. K.-L. \& Yanai, T. Entangled quantum electronic wavefunctions of the $\mathrm{Mn}_{4} \mathrm{CaO}_{5}$ cluster in photosystem II. Nat Chem 5, 660-666 (2013).

[40] Sharma, S. \& Chan, G. K.-L. Spin-adapted density matrix renormalization group algorithms for quantum chemistry. The Journal of Chemical Physics 136, 124121 (2012). 
[41] Chan, G. K. L. \& Head-Gordon, M. Highly correlated calculations with a polynomial cost algorithm: A study of the density matrix renormalization group. J. Chem. Phys. 116, 4462 (2002).

[42] Zgid, D. \& Nooijen, M. On the spin and symmetry adaptation of the density matrix renormalization group method. J. Chem. Phys. 128, 014107 (2008).

[43] Moritz, G., Hess, B. A. \& Reiher, M. Convergence behavior of the density-matrix renormalization group algorithm for optimized orbital orderings. J. Chem. Phys. 122, 024107 (2005).

[44] Kurashige, Y. \& Yanai, T. High-performance ab initio density matrix renormalization group method: applicability to large-scale multireference problems for metal compounds. J. Chem. Phys. 130, 234114 (2009).

[45] Legeza, Ö., Röder, J. \& Hess, B. A. QC-DMRG study of the ionic-neutral curve crossing of LiF. Mol. Phys. 101, 2019 (2003).

[46] Marti, K. H., Ondik, I. M., Mortise, G. \& Reiher, M. Density matrix renormalization group calculations on relative energies of transition metal complexes and clusters. The Journal of Chemical Physics 128, 014104 (2008).

[47] Mayerle, J. J., Denmark, S. E., DePamphilis, B. V., Ibers, J. A. \& Holm, R. H. Synthetic analogs of the active sites of iron-sulfur proteins. XI. Synthesis and properties of complexes containing the iron sulfide $\mathrm{Fe}_{2} \mathrm{~S}_{2}$ core and the structures of bis[o-xylyl- $\alpha, \alpha$-dithiolato- $\mu$-sulfido-ferrate(III)] and bis[p-tolylthiolato- $\mu$-sulfido-ferrate(III)] dianions. Journal of the American Chemical Society 97, 1032-1045 (1975).

[48] Orme-Johnson, W. H. Iron-Sulfur Proteins: Structure and Function. Annual Review of Biochemistry 42, 159-204 (1973).

[49] Venkateswara Rao, P. \& Holm, R. H. Synthetic analogues of the active sites of iron sulfur proteins. Chemical Reviews 104, 527-560 (2003).

[50] Noodleman, L., Case, D., Mouesca, J.-M. \& Lamotte, B. Valence electron delocalization in polynuclear iron-sulfur clusters. JBIC Journal of Biological Inorganic Chemistry 1, 177-182 (1996).

[51] Gillum, W. O., Frankel, R. B., Foner, S. \& Holm, R. H. Synthetic analogues of the active sites of iron-sulfur proteins. XIII. Further electronic structural relationships between the analogues $\left[\mathrm{Fe}_{2} \mathrm{~S}_{2}(\mathrm{SR})_{4}\right]^{2-}$ and the active sites of oxidized $2 \mathrm{Fe}-2 \mathrm{~S}^{*}$ proteins. Inorganic Chemistry 15, 1095-1100 (1976).

[52] Noodleman, L. \& Case, D. A. Density-functional theory of spin polarization and spin coupling in iron-sulfur clusters. Adv. Inorg. Chem 38, 423-470 (1992).

[53] Averill, B. A., Herskovitz, T., Holm, R. H. \& Ibers, J. A. Synthetic analogs of the active sites of iron-sulfur proteins. II. Synthesis and structure of the tetra[mercapto- $\mu 3$-sulfido-iron] clusters, $\left[\mathrm{Fe}_{4} \mathrm{~S}_{4}(\mathrm{SR})_{4}\right]^{2-}$. Journal of the American Chemical Society 95, 3523-3534 (1973).

[54] Noodleman, L., Lovell, T., Liu, T., Himo, F. \& Torres, R. A. Insights into properties and energetics of iron-sulfur proteins from simple clusters to nitrogenase. Current Opinion in Chemical Biology 6, 259-273 (2002).

[55] Shaik, S., Kumar, D., de Visser, S. P., Altun, A. \& Thiel, W. Theoretical Perspective on the Structure and Mechanism of Cytochrome P450 Enzymes. Chemical Reviews 105, 2279-2328 (2005).

[56] Wodtke, A. M., Tully, J. C. \& Auerbach, D. J. Electronically non-adiabatic interactions of molecules at metal surfaces: Can we trust the BornOppenheimer approximation for surface chemistry? International Reviews in Physical Chemistry 23, 513-539 (2004).

[57] Lancaster, K. M. et al. X-ray emission spectroscopy evidences a central carbon in the nitrogenase iron-molybdenum cofactor. Science 334, 974-977 (2011).

[58] Spatzal, T. et al. Evidence for Interstitial Carbon in Nitrogenase FeMo Cofactor. Science 334, 940 (2011). 


\section{Supplementary Information:}

[2Fe-2S]: geometry and orbitals, DMRG calculations, local charge and spin, model Hamiltonians [4Fe-4S]: geometry and orbitals, DMRG calculations, local charge and spin, model Hamiltonians Tables S1-S12

Figures S1-S10

Correspondence and requests for materials should be addressed to G.K.C. 


\title{
Supplementary Information
}

\author{
Sandeep Sharma, Kantharuban Sivalingam, Frank Neese, Garnet Kin-Lic Chan
}

\section{Contents}

I. $[2 \mathrm{Fe}-2 \mathrm{~S}]$ complexes 22

$\begin{array}{ll}\text { A. Geometry and orbitals } & 2\end{array}$

$\begin{array}{ll}\text { B. DMRG calculations } & 3\end{array}$

1. Active spaces 3

2. Energy convergence $\quad 5$

3. Assessment of active space $\quad 6$

$\begin{array}{ll}\text { C. Local charge and spin } & 7\end{array}$

1. Asymmetry in the $\left[\mathrm{Fe}_{2} \mathrm{~S}_{2}\left(\mathrm{SCH}_{3}\right)_{4}\right]^{3-}$ complex 8

D. Model Hamiltonian for the $\left[\mathrm{Fe}_{2} \mathrm{~S}_{2}\left(\mathrm{SCH}_{3}\right)_{4}\right]^{3-}$ dimer 9

$\begin{array}{ll}\text { II. } 4 \mathrm{Fe}-4 \mathrm{~S} \text { cluster } & 15\end{array}$

$\begin{array}{ll}\text { A. Geometry and orbitals } & 15\end{array}$

$\begin{array}{ll}\text { B. DMRG calculations } & 16\end{array}$

$\begin{array}{ll}\text { 1. Active spaces } & 16\end{array}$

2. Energy convergence 16

$\begin{array}{ll}\text { C. Local charge and spin } & 17\end{array}$

D. Model Hamiltonian for pairing and unequal exchange in the [4Fe-4S] cluster 17

1. Single orbital Anderson model 17

2. Multi-orbital Anderson model 20 


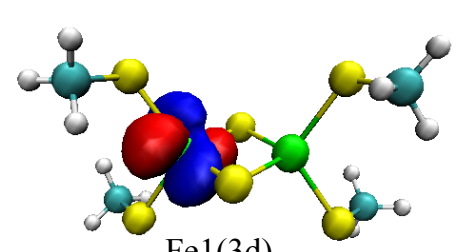

$\mathrm{Fe} 1(3 \mathrm{~d})$

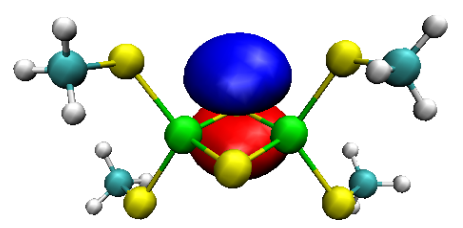

$\mathrm{S} 2\left(3 \mathrm{p}_{0}\right)$
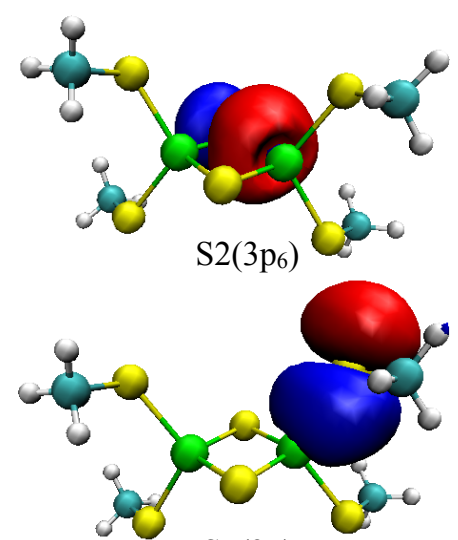

S7(3p)

Supplementary Figure 1: Orbitals in the active space of the $[2 \mathrm{Fe}-2 \mathrm{~S}]$ dimers.

References

\section{I. [2FE-2S] COMPLEXES}

\section{A. Geometry and orbitals}

We used a [2Fe-2S] dimer obtained from the complex of Mayerle et al [1] by substituting the four terminal toluene groups with methyl groups. For the $\left[\mathrm{Fe}_{2} \mathrm{~S}_{2}\left(\mathrm{SCH}_{3}\right)_{4}\right]^{2-}$ complex, the geometry was derived from the experimental structure reported in [1], as shown in Supplementary Table 1. For the $\left[\mathrm{Fe}_{2} \mathrm{~S}_{2}\left(\mathrm{SCH}_{3}\right)_{4}\right]^{3-}$ complex, we considered two geometries: (i) the geometry in Supplementary Table 1 (the same geometry as the $\left[\mathrm{Fe}_{2} \mathrm{~S}_{2}\left(\mathrm{SCH}_{3}\right)_{4}\right]^{2-}$ complex), and (ii) a relaxed geometry, shown in Supplementary Table 2. The relaxed geometry was obtained from a broken-symmetry DFT calculation on the $S_{z}=1 / 2$ state, using the BP86 functional and a split-valence with polarization (SVP) basis set [2] (denoted BP86/SVP) as implemented in Orca[3]. As seen from the table, in the relaxed geometry the dimer becomes slightly asymmetric, with the bridging $\mathrm{S}$ atoms attracted towards one of the $\mathrm{Fe}$ atoms.

To generate the active space for the DMRG calculations, we performed an unrestricted DFT BP86/SVP calculation for the high spin $\left(S_{z}=5\right)$ state. The alpha occupied and unoccupied orbitals were then separately localized ("split-localized") [4] using the Pipek-Mezey algorithm [5]. From the localized orbitals, iron 3d, 4s, 4d and sulfur $3 p$ orbitals were identified by visual inspection. Some of these orbitals are shown in Supplementary Figure 1. 
Supplementary Table 1: Coordinates (in $\AA$ ) of the $\left[\mathrm{Fe}_{2} \mathrm{~S}_{2}\left(\mathrm{SCH}_{3}\right)_{4}\right]^{2-}$ and unrelaxed $\left[\mathrm{Fe}_{2} \mathrm{~S}_{2}\left(\mathrm{SCH}_{3}\right)_{4}\right]^{3-}$ model complexes.

\begin{tabular}{|c|c|c|c|c|}
\hline & & $\mathrm{x}$ & $\mathrm{y}$ & $\mathrm{z}$ \\
\hline 1 & $\mathrm{Fe}$ & 5.22 & 1.05 & -7.95 \\
\hline 2 & $S$ & 3.86 & -0.28 & -9.06 \\
\hline 3 & S & 5.00 & 0.95 & -5.66 \\
\hline 4 & S & 4.77 & 3.18 & -8.74 \\
\hline 5 & $\mathrm{~S}$ & 7.23 & 0.28 & -8.38 \\
\hline 6 & $\mathrm{Fe}$ & 5.88 & -1.05 & -9.49 \\
\hline 7 & $\mathrm{~S}$ & 6.10 & -0.95 & -11.79 \\
\hline 8 & $\mathrm{~S}$ & 6.33 & -3.18 & -8.71 \\
\hline 9 & $\mathrm{C}$ & 6.00 & 4.34 & -8.17 \\
\hline 10 & $\mathrm{H}$ & 6.46 & 4.81 & -9.01 \\
\hline 11 & $\mathrm{H}$ & 5.53 & 5.08 & -7.55 \\
\hline 12 & $\mathrm{H}$ & 6.74 & 3.82 & -7.60 \\
\hline 13 & $\mathrm{C}$ & 3.33 & 1.31 & -5.18 \\
\hline 14 & $\mathrm{H}$ & 2.71 & 0.46 & -5.37 \\
\hline 15 & $\mathrm{H}$ & 3.30 & 1.54 & -4.13 \\
\hline 16 & $\mathrm{H}$ & 2.97 & 2.15 & -5.73 \\
\hline 17 & C & 5.10 & -4.34 & -9.28 \\
\hline 18 & $\mathrm{H}$ & 5.56 & -5.05 & -9.93 \\
\hline 19 & $\mathrm{H}$ & 4.67 & -4.84 & -8.44 \\
\hline 20 & $\mathrm{H}$ & 4.34 & -3.81 & -9.81 \\
\hline 21 & $\mathrm{C}$ & 7.77 & -1.31 & -12.27 \\
\hline 22 & $\mathrm{H}$ & 7.84 & -1.35 & -13.34 \\
\hline 23 & $\mathrm{H}$ & 8.42 & -0.54 & -11.90 \\
\hline 24 & $\mathrm{H}$ & 8.06 & -2.25 & -11.86 \\
\hline
\end{tabular}

\section{B. DMRG calculations}

1. Active spaces

Four types of active space DMRG calculations (labelled (1)-(4)) were performed on the $\left[\mathrm{Fe}_{2} \mathrm{~S}_{2}\left(\mathrm{SCH}_{3}\right)_{4}\right]^{2-}$ complex to assess the effect of active space choice. For the $\left[\mathrm{Fe}_{2} \mathrm{~S}_{2}\left(\mathrm{SCH}_{3}\right)_{4}\right]^{3-}$ complex, we used only active spaces (1) and (2), following the analysis in section I B 3.

All DMRG calculations were spin-adapted, using the BLOCK code as described in Ref. [6]. Thus all states obtained are eigenfunctions of $S_{z}$ and $S^{2}$, and $M$ refers to the number of spin-adapted renormalized states (the tensor link dimension in the one-dimensional tensor network underlying the DMRG) which corresponds to effec- 
Supplementary Table 2: Coordinates (in $\AA$ ) of the relaxed $\left[\mathrm{Fe}_{2} \mathrm{~S}_{2}\left(\mathrm{SCH}_{3}\right)_{4}\right]^{3-}$ model complex.

\begin{tabular}{|c|c|c|c|}
\hline & $\mathrm{x}$ & y & $\mathrm{z}$ \\
\hline $1 \mathrm{Fe}$ & Fe 5.48 & 1.15 & -8.03 \\
\hline $2 \mathrm{~S}$ & $\begin{array}{ll}S \quad 4.05\end{array}$ & -0.61 & -8.75 \\
\hline $3 \mathrm{~S}$ & $\begin{array}{ll}\text { S } 5.47\end{array}$ & 1.25 & -5.58 \\
\hline $4 \mathrm{~S}$ & $\begin{array}{ll}S \quad 4.63 \\
\end{array}$ & 3.28 & -8.77 \\
\hline $5 \quad \mathrm{~S}$ & $\begin{array}{ll}S \quad 7.49\end{array}$ & 0.42 & -9.04 \\
\hline $6 \mathrm{Fe}$ & $\mathrm{Fe} 6.04$ & -1.22 & -9.63 \\
\hline $7 \quad \mathrm{~S}$ & S 5.75 & -1.50 & -12.05 \\
\hline $8 \mathrm{~S}$ & S 6.86 & -3.41 & -8.86 \\
\hline $9 \quad \mathrm{C}$ & C 5.51 & 4.45 & -7.51 \\
\hline $10 \mathrm{H}$ & H 6.49 & 4.83 & -7.92 \\
\hline $11 \mathrm{H}$ & $\begin{array}{ll}\text { H } 4.87 \\
\end{array}$ & 5.33 & -7.25 \\
\hline $12 \mathrm{H}$ & H 5.72 & 3.84 & -6.59 \\
\hline $13 \mathrm{C}$ & C 3.60 & 1.70 & -5.54 \\
\hline $14 \mathrm{H}$ & $\begin{array}{ll}\text { H } 3.01 \\
\end{array}$ & 0.80 & -5.82 \\
\hline $15 \mathrm{H}$ & $\begin{array}{ll}\text { H } 3.28 \\
\end{array}$ & 2.06 & -4.52 \\
\hline $16 \mathrm{H}$ & $\begin{array}{ll}\mathrm{H} & 3.42\end{array}$ & 2.48 & -6.31 \\
\hline $17 \mathrm{C}$ & C 5.21 & -4.22 & -9.46 \\
\hline $18 \mathrm{H}$ & $\begin{array}{ll}\text { H } 5.10\end{array}$ & -4.01 & -10.55 \\
\hline $19 \mathrm{H}$ & H 5.21 & -5.32 & -9.26 \\
\hline $20 \mathrm{H}$ & $\begin{array}{ll}\text { H } 4.37\end{array}$ & -3.72 & -8.93 \\
\hline $21 \mathrm{C}$ & С 7.63 & -1.85 & -12.24 \\
\hline $22 \mathrm{H}$ & $\begin{array}{ll}\text { H } 7.90 \\
\end{array}$ & -2.06 & -13.31 \\
\hline $23 \mathrm{H}$ & $\begin{array}{ll}\mathrm{H} \quad 8.20 \\
\end{array}$ & -0.96 & -11.86 \\
\hline $24 \mathrm{H}$ & $\begin{array}{l}\mathrm{H} \quad 7.89 \\
\end{array}$ & -2.72 & -11.59 \\
\hline
\end{tabular}

tively twice the number of non-spin-adapted renormalized states in a standard DMRG calculation [6]. The 4 types of DMRG calculations were:

1. DMRG-CI on a (30e, 20o) active space, with a maximum of $M=3500$ renormalized states. The 20 orbitals included Fe 3d, bridging S 3p, and one 3p orbital per terminal ligand $\mathrm{S}$ atom. This corresponds to a minimal full valence active space. For rapid convergence of the DMRG energy, the orbitals were ordered as follows: S4(3p), S3(3p), Fe1(3d), Fe1(3d), Fe1(3d), Fe1(3d), Fe1(3d), S2(3p 1 ), S5(3p 1 ), S2(3p 0 ), S5(3p 0 ), S2(3p $\left.\mathrm{p}_{6}\right)$, S5(3p $\left.p_{6}\right), F e 6(3 d), F e 6(3 d), F e 6(3 d), F e 6(3 d), F e 6(3 d), S 7(3 p), S 8(3 p)$, where the atom labels correspond to the labels in Supplementary Tables 1 and 2, and Figure ??, and the subscript on S 3p orbitals is the index of the atom they are pointing towards, with the exception that the subscript 0 means that it is pointing in the 
up-down direction as shown in Figure 1.

2. DMRG-CI on a (30e, 32o) active space, with a maximum of $M=4500$ renormalized states. The 30 orbitals include $\mathrm{Fe} 4 \mathrm{~d}$ and $\mathrm{Fe} 4 \mathrm{~s}$ orbitals in addition to the 20 described in active space (1). The $\mathrm{Fe} 4 \mathrm{~d}$ and $4 \mathrm{~s}$ orbitals are expected to account for the principal dynamic and orbital relaxation contributions to the energy (i.e. double-shell correlation [7]). The orbitals were ordered as for the 20 orbital active space, with additional Fe $4 \mathrm{~s}$ and $\mathrm{Fe} 4 \mathrm{~d}$ orbitals placed in that order immediately following the $3 \mathrm{~d}$ orbitals of the same Fe atom.

3. DMRG-SCF on the (30e, 20o) active space (cf. active space(1)). The active space orbitals were optimized using a self-consistent cycle. The DMRG calculations in the SCF optimization used $M=2500$ states. Subsequently a final calculation with $M=3500$ states, using the fixed optimized orbitals, was performed. The same ordering as in active space (1) was used.

4. DMRG-SCF on the (30e, 32o) active space (cf. active space (2)). Again, the DMRG calculations in the SCF optimization were performed with $M=2500$. Subsequently a final calculation with $M=4500$ states, using the fixed optimized orbitals, was performed. The same ordering as in active space (2) was used.

\section{Energy convergence}

The DMRG energies and discarded weights at different values of $M$ can be used to extrapolate the energy to the $M=\infty$ (FCI) result, which corresponds to zero discarded weight. This also provides error estimates for the DMRG energy [8]. Extrapolations for state-specific DMRG-CI (active space 2) calculations are shown in Supplementary Table 3 and Supplementary Figure 2. We find that the extrapolated (30e, 32o) singlet and triplet relative energies are converged to within $0.1 \mathrm{~m} E_{h}$ of the FCI energy.

For the $[2 \mathrm{Fe}-2 \mathrm{~S}]$ spectrum calculations, we computed the lowest 10 states in each spin-sector using a stateaveraged DMRG calculation. Although the energies are not as well converged as for the state-specific calculations, the residual errors do not qualitatively affect the spectrum or the conclusions of our analysis. 
Supplementary Table 3: DMRG energy in $E_{h}$ versus the discarded weight of the singlet and triplet states of the $\left[\mathrm{Fe}_{2} \mathrm{~S}_{2}\left(\mathrm{SCH}_{3}\right)_{4}\right]^{-2}$ cluster (active space (2), (30e, 32o)).

\begin{tabular}{cccccc}
\hline \hline & \multicolumn{2}{c}{ Singlet } & & \multicolumn{2}{c}{ Triplet } \\
\cline { 2 - 3 } \cline { 5 - 6 } $\mathrm{M}$ & Discarded weight & Energy & & Discarded weight & Energy \\
\hline 1500 & $2.45 \times 10^{-5}$ & $-5,104.138933$ & & $2.54 \times 10^{-5}$ & $-5,104.135801$ \\
2500 & $1.14 \times 10^{-5}$ & $-5,104.139978$ & & $1.23 \times 10^{-5}$ & $-5,104.137651$ \\
3500 & $5.63 \times 10^{-6}$ & $-5,104.140297$ & & $8.54 \times 10^{-6}$ & $-5,104.138315$ \\
4500 & $3.60 \times 10^{-6}$ & $-5,104.140426$ & & $6.03 \times 10^{-6}$ & $-5,104.138616$ \\
$\infty$ & & $-5,104.140718$ & & $-5,104.139510$ \\
\hline
\end{tabular}

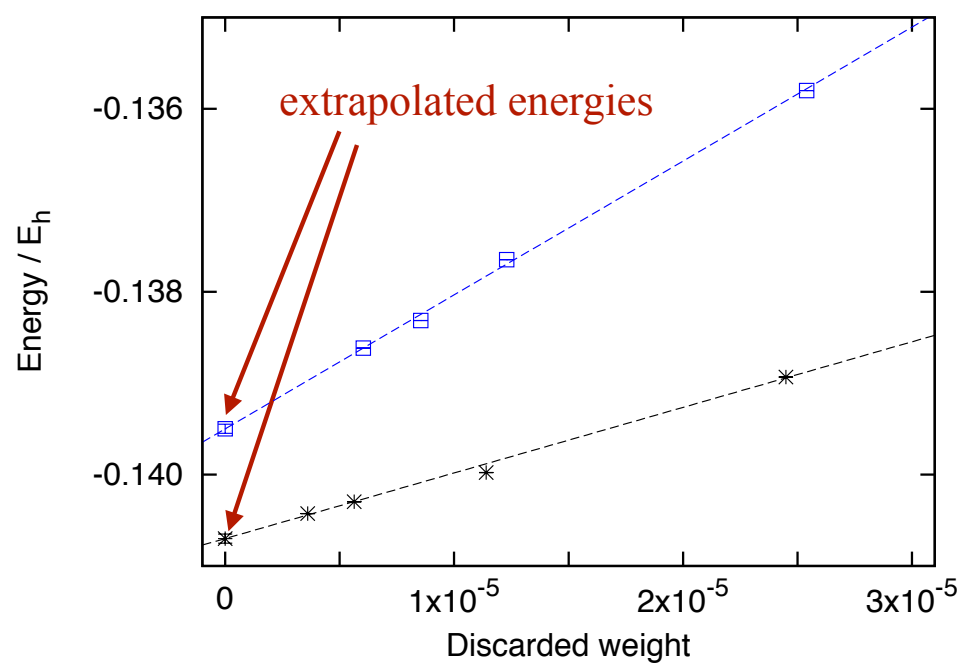

Supplementary Figure 2: DMRG energy $(\mathrm{E}+5104.0)$ in $E_{h}$ of the singlet and triplet states versus the discarded weight of the $\left[\mathrm{Fe}_{2} \mathrm{~S}_{2}\left(\mathrm{SCH}_{3}\right)_{4}\right]^{-2}$ cluster (active space (2), (30e, 32o). The black crosses and the blue dots are respectively the DMRG singlet and triplet state energies and the corresponding lines are the best fit straight lines which are extrapolated to zero discarded weight to obtain an estimated FCI energy.

\section{Assessment of active space}

From the singlet-triplet gap in the active spaces (1)-(4), shown in Supplementary Table 4, we can assess the effect of the active space choice on the computed energy levels. We first observe that all 4 active spaces agree closely; even the minimal valence active space yields a reasonable gap. This is because the principle exchange pathway leading to the singlet-triplet gap is via the bridging $\mathrm{S} 3 \mathrm{p}$ ligand orbitals, which are included in the minimal active space. The effect of double-shell correlation in the larger active spaces is to increase the gap by less than $1.0 \mathrm{~m} E_{h}$, 
Supplementary Table 4: The singlet-triplet gap of the $\left[\mathrm{Fe}_{2} \mathrm{~S}_{2}\left(\mathrm{SCH}_{3}\right)_{4}\right]^{2-}$ complex obtained in active spaces (1)-(4).

\begin{tabular}{ccc}
\hline \hline Method & Active Space & $\mathrm{Gap} / \mathrm{m} E_{h}$ \\
\hline DMRG-CI & $(30 \mathrm{e}, 20 \mathrm{o})$ & 1.5 \\
DMRG-CI & $(30 \mathrm{e}, 32 \mathrm{o})$ & 2.1 \\
DMRG-SCF & $(30 \mathrm{e}, 20 \mathrm{o})$ & 1.2 \\
DMRG-SCF & $(30 \mathrm{e}, 32 \mathrm{o})$ & 2.0 \\
\hline \hline
\end{tabular}

while the effect of orbital optimization is very small $\left(0.1 \mathrm{~m} E_{h}\right.$ in the larger active space). We take active space (2) (double shell correlation but no orbital optimization) as a practical compromise between accuracy and efficiency.

\section{Local charge and spin}

To identify the distribution of electrons, we have computed local populations on the atoms. For atom $A$, the local population $N_{A}$ is

$$
N_{A}=\sum_{i \in A} n_{i}
$$

where $n_{i}$ is the number operator of localized orbital $i$ on the $A$.

Further, since our states are eigenstates of $S^{2}$, there is no spin-density in the singlet states. Thus, we have computed local spins and spin-correlation functions to characterize the electronic structure. The spin-correlation function between atoms $A$ and $B,\left\langle S_{A} \cdot S_{B}\right\rangle$, is defined as [9-11]

$$
\begin{aligned}
S_{A} \cdot S_{B} & =\sum_{\alpha} S_{A}^{\alpha} S_{B}^{\alpha} \\
S_{A}^{\alpha} & =\sum_{i \in A} s_{i}^{\alpha}
\end{aligned}
$$

where $\alpha \in\{x, y, z\}$. The local total spin on atom $A$ is defined as $\left\langle S_{A} \cdot S_{A}\right\rangle$.

Supplementary Tables 6-9 show the relative energies, local populations, spins, and spin-correlation functions for the $[2 \mathrm{Fe}-2 \mathrm{~S}]$ complexes. All the relative energies reported in the tables are calculated using active space (2). For both geometries state averaged DMRG calculations are performed for the first 10 states with a largest M of 4500. In the case of unrelaxed geometries three sweeps with $M=4500$ were performed and then its value was 
Supplementary Table 5: The local population, total spin, and spin-correlation functions in the lowest spin states of the $\left[\mathrm{Fe}_{2} \mathrm{~S}_{2}\left(\mathrm{SCH}_{3}\right)_{4}\right]^{2-}$ complex, using active space (2). Note $\left\langle N_{2}\right\rangle=\left\langle N_{1}\right\rangle$ and $\left\langle S_{2}^{2}\right\rangle=\left\langle S_{1}^{2}\right\rangle$. One orbitals of the Fe

\begin{tabular}{cccc}
\hline \hline Dimer $S$ & $\left\langle N_{1}\right\rangle$ & $\left\langle S_{1}^{2}\right\rangle$ & $\left\langle S_{1} \cdot S_{2}\right\rangle$ \\
\hline 0 & 6.18 & 5.47 & -4.92 \\
1 & 6.17 & 5.47 & -4.22 \\
2 & 6.17 & 5.49 & -2.84 \\
3 & 6.17 & 5.51 & -0.79 \\
4 & 6.16 & 5.54 & 1.84 \\
5 & 6.13 & 5.74 & 3.74 \\
\hline \hline
\end{tabular}

reduced in steps of 1000 down to $M=1500$ to generate reliable extrapolated energies. We find that even though the extrapolation process improves the absolute energies, the energy differences reported in the tables are relatively unchanged. We expect to see the same trend for the relaxed geometries and thus forego the expensive extrapolation step to report the relative DMRG energies calculated with $\mathrm{M}=4500$.

In Supplementary Table 6 and Supplementary Table 8 only the 3d, 4s and 4d orbitals of a Fe atom are included in the summations in Equations 1 and 2 to calculate the local electron density and electron spin; whereas in Supplementary Table 7 and Supplementary Table 9 the first 16 orbitals and second 16 orbitals (see orbital ordering in previous section for the orbitals) are included in the summations for first and the second Fe atoms respectively. The ideal $\mathrm{Fe}^{\mathrm{II}}$ and $\mathrm{Fe}^{\mathrm{III}}$ populations are 6 and 5 respectively, while the ideal $S=2$ and $S=5 / 2$ total spins are 6 and 8.75 respectively. We see that the observed local populations and total spins are increased and reduced respectively in the complexes due to the effect of quantum fluctuations, such as delocalization onto adjacent sulfur orbitals. We also see from the spin-correlation functions that the spins progressively move from being anti-aligned to aligned as the the total dimer spin is increased.

1. Asymmetry in the $\left[\mathrm{Fe}_{2} \mathrm{~S}_{2}\left(\mathrm{SCH}_{3}\right)_{4}\right]^{3-}$ complex

The relaxed geometry of the $\left[\mathrm{Fe}_{2} \mathrm{~S}_{2}\left(\mathrm{SCH}_{3}\right)_{4}\right]^{3-}$ complex is slightly asymmetric. The local populations and spins in Supplementary Table 8 and 9 show the effect of this asymmetry on the electronic structure. As observed in Supplementary Table 8 the asymmetry in the Fe atoms appears rather small, amounting to up to 0.05 electron 
units in the population, and 0.2 spin units in the local spin. But when we compare the local spins shown in Supplementary Table 9, the asymmetry is much larger with differences between the local spins as large as 2.4 in some cases. The difference in asymmetry between the two tables points to the fact that the asymmetry is largely due to the difference in the $S 3 p$ orbitals.

\section{Model Hamiltonian for the $\left[\mathrm{Fe}_{2} \mathrm{~S}_{2}\left(\mathrm{SCH}_{3}\right)_{4}\right]^{3-}$ dimer}

The energy levels of the HDE model for the $\left[\mathrm{Fe}_{2} \mathrm{~S}_{2}\left(\mathrm{SCH}_{3}\right)_{4}\right]^{3-}$ mixed valence complex, as derived by Noodleman and Baerends, are given by

$$
E(S)=2 J S_{1} \cdot S_{2} \pm B(S+1 / 2)
$$

As demonstrated in the main text, the HDE energy levels do not fit the ab-initio DMRG spectrum well because of the assumptions used to derive Eq. (3). Before deriving a more complete model that is compatible with the ab-initio spectrum, we briefly recall how Eq. (3) is obtained from Anderson's analysis of double exchange [12-14].

We first consider an oxidized complex with two ferric ions (with spins $S_{1}=5 / 2, S_{2}=5 / 2$ ) as a "base" system. The extra electron in the reduced dimer is added to this base system, where it hops between a pair of local orbitals on each of the ions. Denoting the creation (annihilation) operators for the local orbitals on the first (second) ions by $c_{1}^{(\dagger)}, c_{2}^{(\dagger)}$ respectively, and the spin of the electron as $s_{1}, s_{2}$ respectively, Anderson's analysis[12] leads to a Hamiltonian of the form

$$
H=J\left(S_{1} \cdot S_{2}+S_{1} \cdot s_{2}+S_{2} \cdot s_{1}\right)+\sum_{\sigma=\uparrow, \downarrow} \beta\left(c_{1 \sigma}^{\dagger} c_{2 \sigma}+c_{2 \sigma}^{\dagger} c_{1 \sigma}\right)
$$

where the Hamiltonian is to be solved in the Hilbert space where the hopping electron is always anti-aligned with the spin of the ferric ion on which it is currently residing. The terms in $H$ have the following meaning:

1. The first corresponds to Heisenberg exchange coupling between spins on the two ions (the "base" ferric spins and the extra spin of the hopping electron). 
2. The second describes the effective hopping of the electron between the two ferric ions (the sum over $\sigma$ is a summation over electron spin).

The eigenvalues of the Anderson Hamiltonian may be determined analytically to be the HDE energy levels in Eq. (3), where $B=\beta /(2 S+1), S=S_{1}=S_{2}$.

As argued in the main text, the most commonly used version of the HDE model breaks down in the $\left[\mathrm{Fe}_{2} \mathrm{~S}_{2}\left(\mathrm{SCH}_{3}\right)_{4}\right]^{3-}$ dimer because it assumes that there is a single pair of d orbitals on the ferric ions that participates in the hopping process. This assumption is valid if the double exchange splitting $B(S+1 / 2)$ is much smaller than the ligand-field splitting $\Delta$. However, this is clearly not the case for Fe ions with tetrahedral coordination which is typically associated with weak ligand fields. Instead, all 5 pairs of $d$ orbitals participate in the hopping at low energies.

We can extend Anderson's double exchange Hamiltonian to multi-orbital double exchange. We label each of the 5 local $\mathrm{d}$ orbitals by index $i$. This gives

$$
H=\sum_{i j} J_{i j} s_{1 i} \cdot s_{2 j}+\sum_{i \sigma}\left[\beta_{i}\left(c_{1 i \sigma}^{\dagger} c_{2 i \sigma}+c_{2 i \sigma}^{\dagger} c_{1 i \sigma}\right)+\Delta_{i}\left(c_{1 i \sigma}^{\dagger} c_{1 i \sigma}+c_{2 i \sigma}^{\dagger} c_{2 i \sigma}\right)\right]
$$

where we once again restrict ourselves to states where the hopping electron is strictly antiferromagnetically aligned to the base spins (the base spins are all ferromagnetically aligned). The additional $\Delta_{i}$ term gives the ligand field splitting of the orbitals. The above form has a very large number of parameters from the general exchange couplings $J_{i j}$. However, in the limiting case where all spins are aligned on each Fe atom (e.g. as in the oxidized dimer), then it is sufficient to consider an exchange term of the form $\sum_{i} J_{i}^{\prime} s_{1 i} \cdot s_{2 i}$ since the interaction of any spin on a given Fe atom, with any spin on the other Fe atom is the same, i.e. $s_{11} \cdot s_{21}=s_{1 i} \cdot s_{21}$ for all $i$, and $J_{i}=\sum_{j} J_{i j}$. Keeping this form for the reduced dimer, we arrive at an Anderson Hamiltonian

$$
H=\sum_{i} J_{i} s_{1 i} \cdot s_{2 i}+\sum_{i \sigma}\left[\beta_{i}\left(c_{1 i \sigma}^{\dagger} c_{2 i \sigma}+c_{2 i \sigma}^{\dagger} c_{1 i \sigma}\right)+\Delta_{i}\left(c_{1 i \sigma}^{\dagger} c_{1 i \sigma}+c_{2 i \sigma}^{\dagger} c_{2 i \sigma}\right)\right]
$$

which is the one used in the main text. Note that when solving for the eigenvalues of the Hamiltonian, we restrict each ion to have at most one additional electron (i.e. the lowest oxidation state is ferrous). 


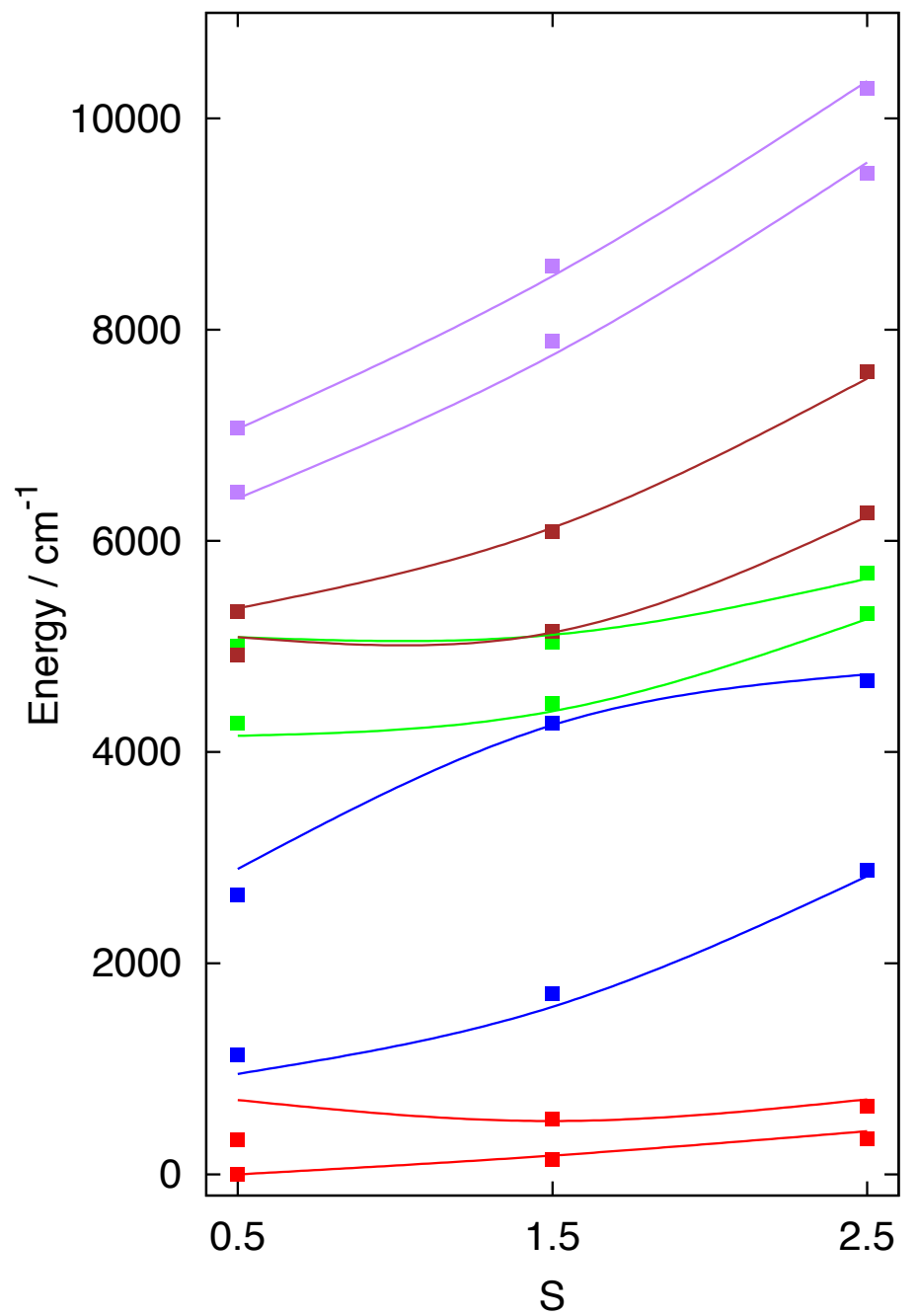

Supplementary Figure 3: Fit of first 10 states each with spins from $S=1 / 2$ to $5 / 2$ using the multi-orbital Anderson model (see Equation 6).

The above multi-orbital Hamiltonian does not admit an analytic solution. However, we can solve for its eigenvalues and eigenvectors numerically. We have written a code to do this which works with an arbitrary number of base spins and hopping electrons and which we use also with the [4Fe-4S] Hamiltonian discussed later. The code is made efficient by working in the basis where the hopping electron is strictly anti-ferromagnetically aligned to the base spins. The hopping matrix elements in this basis can be calculated using Clebsch-Gordan coefficients, similar to Anderson's original work [12]. The code can be downloaded with this paper. 
A direct fit of the multi-orbital Hamiltonian to the DMRG ab-initio levels yields the parameters in Supplementary Table 10 and the levels in Supplementary Figure 3. As we can see the fit is very good; the r.m.s. error is only about $60 \mathrm{~cm}^{-1}$. Further it is very robust: out of 12 random initial starting fits, all fits either converged to the same physical solution shown (to within the standard deviation in the parameters), or attempted to find unphysical solutions with negative parameters. Overall, this demonstrates that the multi-orbital Hamiltonian indeed captures the essential low-energy physics of the $\left[\mathrm{Fe}_{2} \mathrm{~S}_{2}\left(\mathrm{SCH}_{3}\right)_{4}\right]^{3-}$ complex. Note that the plots in Fig. 3 are for dimer spins $S=1 / 2,3 / 2,5 / 2$ only. This is because for the higher dimer spins, some of the excited states appear to have acquire d-d transition character, i.e. the Fe ions are not truly high spin. This can be seen, for example in states 8 and 10 for $S=7 / 2$ in Supplementary Table 6. Such states probably exist in the weak-shoulder region below 10000 $\mathrm{cm}^{-1}$ in the low-temperature absorption spectrum of ferredoxins [15], and cannot be described with the model Hamiltonians we are using.

As emphasized in the text, the multi-orbital Hamiltonian is not equivalent to the simple multi-pair generalization of the HDE model. This would correspond to extending the HDE energy levels in Eq. (3) to 5 separate pairs of levels arising from each of the pair of d orbitals,

$$
E_{i}(S)=\Delta_{i}+2 J_{i} S_{1} \cdot S_{2} \pm B_{i}\left(S_{i}+1 / 2\right)
$$

where the subscript $i$ denotes the pair involved in the hopping. This multi-pair HDE model in fact has the same number of parameters as the multi-orbital Hamiltonian (6) itself. However, as seen in the Supplementary Figure ?? in the main text, the naive form does not fit the ab-initio DMRG results. Multi-orbital double exchange cannot generally be viewed simply as the sum of individual orbital double-exchange processes. In particular, this means that to be precise we should not characterize double exchange by an effective $B$ parameter as in the HDE model, but rather by hopping integrals, $\beta$.

Further support for the multi-orbital nature of the double exchange is obtained from density difference plots, shown in Supplementary Figure 5. These plots are obtained as the difference density between different singlet states. If a single well-defined d orbital pair were to give rise to a pair of states, then we would expect the density difference to resemble a density associated with a particular $d$ pair. However, we find that, aside from the lowest two 


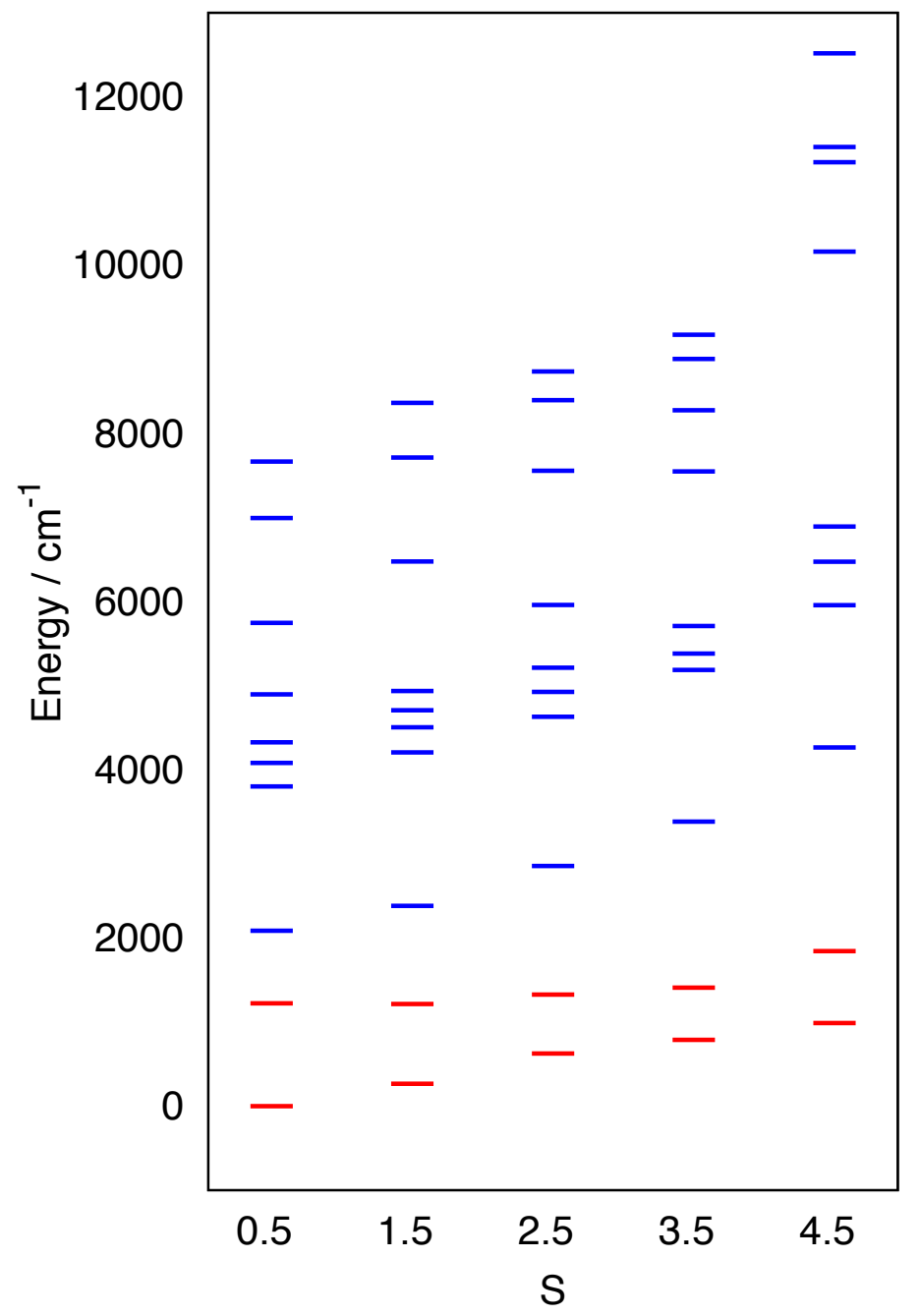

Supplementary Figure 4: The computed DMRG energy levels at the relaxed $\left[\mathrm{Fe}_{2} \mathrm{~S}_{2}\left(\mathrm{SCH}_{3}\right)_{4}\right]^{3-}$ dimer geometry.

pair of states (which appear to have some $e_{g}$ parentage), the remaining density differences involve contributions from all sets of d orbital densities.

We now briefly discuss the effect of geometry relaxation on the $\left[\mathrm{Fe}_{2} \mathrm{~S}_{2}\left(\mathrm{SCH}_{3}\right)_{4}\right]^{3-}$ dimer energy levels. At the relaxed geometry, some localization of the charge occurs. The computed DMRG energy levels at the relaxed geometry are shown in Fig. 4. We find that our main observations are unchanged: there is little separation between the lowest two and higher energy levels, and the gap between the lowest two levels does not monotonically 

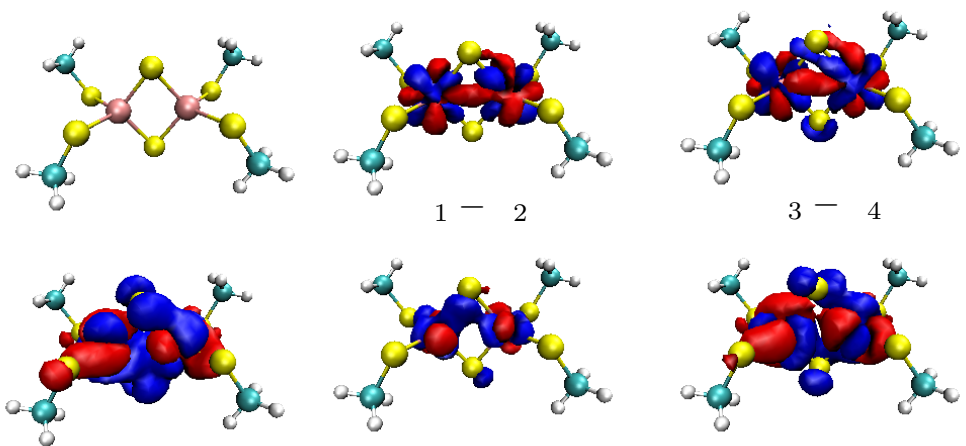

$$
5-6
$$

$$
5-7
$$
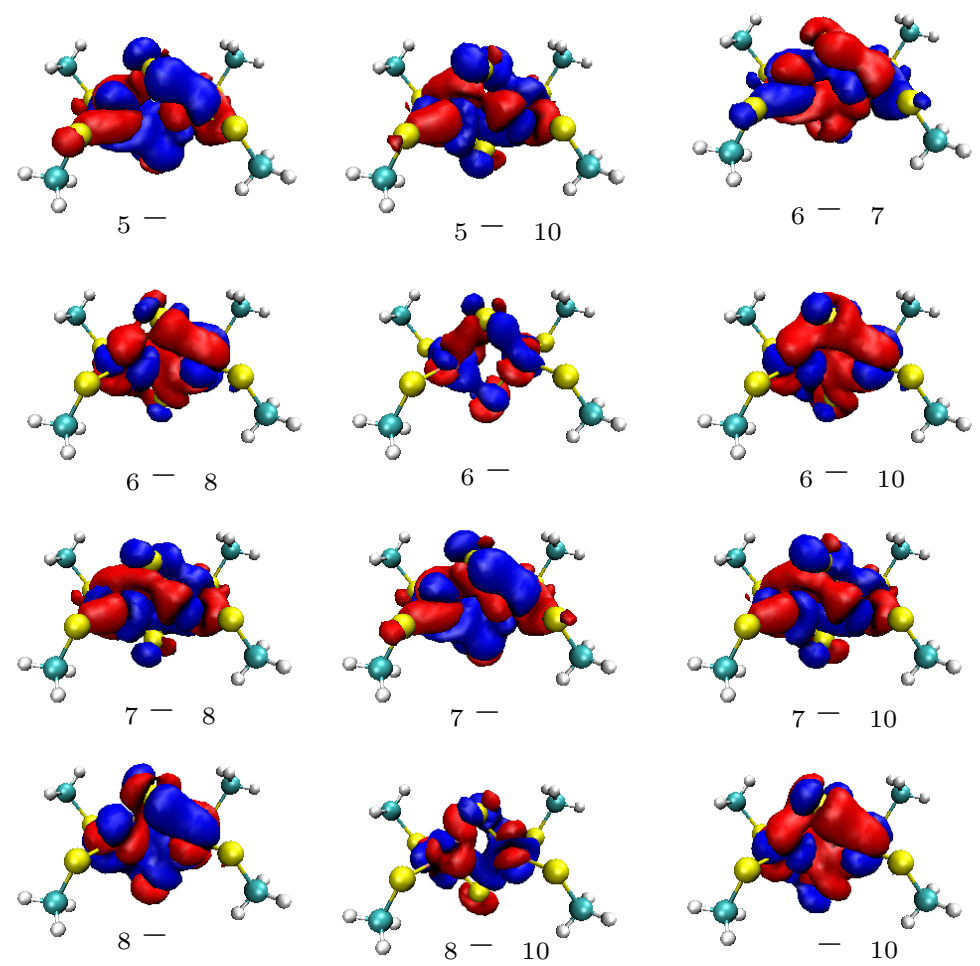

Supplementary Figure 5: Density differences between sets of doublet states in the $\left[\mathrm{Fe}_{2} \mathrm{~S}_{2}\left(\mathrm{SCH}_{3}\right)_{4}\right]^{3-}$ complex. We notice that besides the lowest two pairs of states, the density difference involves contributions from many d orbitals.

increase as required by the HDE model. We have not computed further relaxation effects from solvent and vibronic coupling, but it is clear from the above that our conclusions about the multi-orbital nature of double exchange and the need for the multi-orbital Anderson model hold quite generally. 


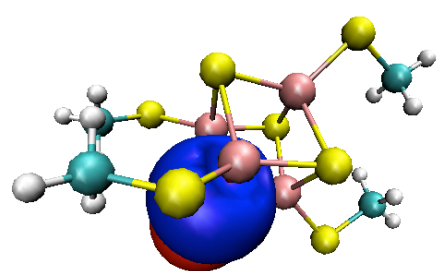

$\mathrm{S} 1(3 \mathrm{p} 5)$

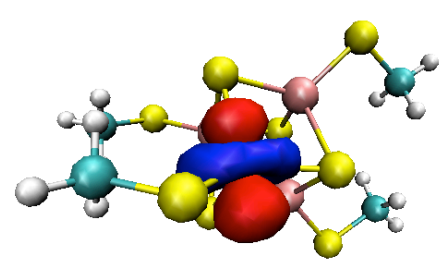

$\mathrm{Fe} 5(3 \mathrm{~d})$

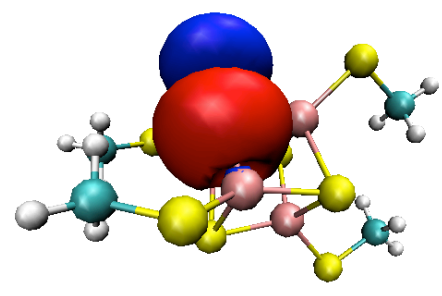

$\mathrm{S} 4\left(3 \mathrm{p}_{5}\right)$

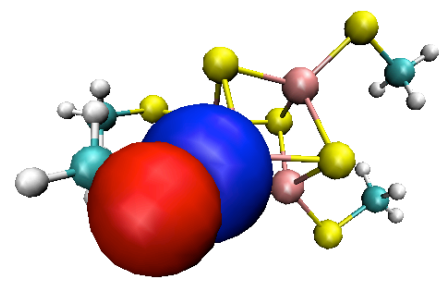

$\mathrm{S} 10\left(3 \mathrm{p}_{5}\right)$

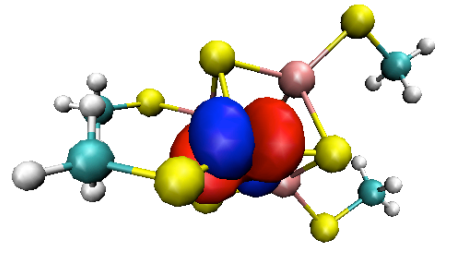

$\mathrm{Fe} 5(3 \mathrm{~d})$

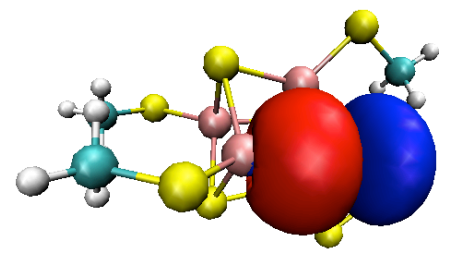

$\mathrm{S} 3\left(3 \mathrm{p}_{5}\right)$

Supplementary Figure 6: Orbitals in the active space of the $\left[\mathrm{Fe}_{4} \mathrm{~S}_{4}\left(\mathrm{SCH}_{3}\right)_{4}\right]^{2-}$ cluster.

\section{4FE-4S CLUSTER}

\section{A. Geometry and orbitals}

For the $[4 \mathrm{Fe}-4 \mathrm{~S}]$ cluster, we computed an optimized BS-DFT geometry using the BP86 functional and a triple zeta valence basis (TZV) basis set [16]. The optimized geometry is shown in Supplementary Table 11.

The active space orbitals are chosen in the same way as in the case of the [2Fe-2S] dimer. An unrestricted DFT BP86/SVP calculation was performed at the optimized geometry for the neutral [4Fe-4S] (all ferric) cluster in the high spin $\left(S_{z}=10\right)$ state. The occupied and unoccupied alpha orbitals were then separately localized using the Pipek-Mezey localization technique. The $20 \mathrm{Fe} 3 \mathrm{~d}$ orbitals, 12 bridging S 3p orbitals, as well as 4 terminal ligand S 3p orbitals that point towards the Fe atoms, were identified by visual inspection. The occupancy of these orbitals in the $\left[\mathrm{Fe}_{4} \mathrm{~S}_{4}\left(\mathrm{SCH}_{3}\right)_{4}\right]^{2-}$ cluster gives an active space of $(54 \mathrm{e}, 36 \mathrm{o})$. Some representative orbitals in the active space are shown in Supplementary Figure 6. 


\section{B. DMRG calculations}

\section{Active spaces}

The DMRG-CI calculation with an active space of $(54 \mathrm{e}, 36 \mathrm{o})$ is performed with a maximum of $M=7500$ spinadapted renormalized states. The 36 orbitals include $20 \mathrm{Fe} 3 \mathrm{~d}$ orbitals, 12 bridging S $3 \mathrm{p}$ orbitals, as well as 4 terminal ligand S 3p orbitals that point towards the Fe atoms. For rapid convergence of DMRG energy these

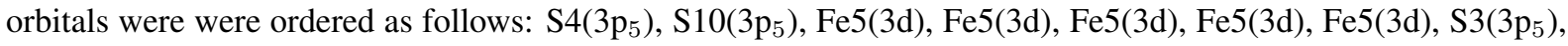
$\mathrm{S} 1\left(3 \mathrm{p}_{5}\right), \mathrm{S} 1\left(3 \mathrm{p}_{6}\right), \mathrm{S} 4\left(3 \mathrm{p}_{6}\right), \mathrm{Fe} 6(3 \mathrm{~d}), \mathrm{Fe} 6(3 \mathrm{~d}), \mathrm{Fe} 6(3 \mathrm{~d}), \mathrm{Fe} 6(3 \mathrm{~d}), \mathrm{Fe} 6(3 \mathrm{~d}), \mathrm{S} 11\left(3 \mathrm{p}_{6}\right), \mathrm{S} 2\left(3 \mathrm{p}_{6}\right), \mathrm{S} 4\left(3 \mathrm{p}_{7}\right), \mathrm{S} 2\left(3 \mathrm{p}_{7}\right)$, $\mathrm{Fe} 7(3 \mathrm{~d}), \mathrm{Fe} 7(3 \mathrm{~d}), \mathrm{Fe} 7(3 \mathrm{~d}), \mathrm{Fe} 7(3 \mathrm{~d}), \mathrm{Fe} 7(3 \mathrm{~d}), \mathrm{S} 3\left(3 \mathrm{p}_{7}\right), \mathrm{S} 9\left(3 \mathrm{p}_{7}\right), \mathrm{S} 3\left(3 \mathrm{p}_{8}\right), \mathrm{S} 1\left(3 \mathrm{p}_{8}\right), \mathrm{Fe} 8(3 \mathrm{~d}), \mathrm{Fe} 8(3 \mathrm{~d}), \mathrm{Fe} 8(3 \mathrm{~d})$, $\mathrm{Fe} 8(3 \mathrm{~d}), \mathrm{Fe} 8(3 \mathrm{~d}), \mathrm{S} 2\left(3 \mathrm{p}_{8}\right), \mathrm{S} 12\left(3 \mathrm{p}_{8}\right)$, where the atom labels correspond to the labels in Supplementary Tables 11, and Supplementary Figure ??, and the subscript on S 3p orbitals is the index of the atom they are pointing towards.

\section{Energy convergence}

As mentioned in the main text of the article (see panel A of Supplementary Figure ??), in a perfect cubane cluster there are three equivalent pairings of the spins of the four Fe atoms to form a singlet ground state. In practice, structural distortion lifts this degeneracy, but the electronic distortion energy is quite small (associated with an energy scale of less than $8 \mathrm{~m} E_{h}$ as seen in 7). This small energy difference can make robust convergence of the DMRG wave function to the true ground state difficult because the wavefunction optimization can get stuck early on in the "wrong" pairing. To expedite the convergence of the wave function towards the correct ground state the Hamiltonian is artificially perturbed so that atoms pairs ( $\mathrm{Fe} 5, \mathrm{Fe} 6)$ and $(\mathrm{Fe} 7, \mathrm{Fe} 8)$ gain a strong tendency to ferromagnetically align. This is done by artificially increasing the exchange integrals between the $3 \mathrm{~d}$ orbitals of the paired up Fe atoms by $0.01 E_{h}$ until the number of renormalized states $M=1600$ is reached. Subsequently this perturbation in the Hamiltonian is decreased to zero over the next few sweeps, and the rest of the DMRG calculation (up to $M=7500$ ) is performed on the unperturbed Hamiltonian.

In fact, the above method of perturbing the Hamiltonian can be used to "converge" the DMRG wavefunction 
towards any of the three pairings shown in Supplementary Figure ??. The ground-state pairing is identified as the one with the lowest energy. The other pairings do not necessarily approximate exact eigenstates of the full Hamiltonian, but rather, are local minima in the parameter space of DMRG. They are a form of "broken-symmetry" DMRG solution, and can be thought of as the best DMRG states that can be obtained with a maximum $M=7500$, when the spin couplings of the various Fe centers is constrained to be non-optimal. The energy differences between these different solutions can be used to estimate the difference in the exchange coupling coefficients of the HDE model hamiltonian (see Section II D).

The DMRG energies and discarded weights at different values of $M$ are used to extrapolate to zero discarded weight, which also gives us the estimated energy errors. This is shown in Supplementary Figure 7. Our estimated error in the total energies is less than $1 \mathrm{~m} E_{h}$.

\section{Local charge and spin}

Local populations and spin correlation functions between different Fe atoms 1-4 can be calculated using the equations 1 and 2 and are given in Supplementary Table 12.

\section{Model Hamiltonian for pairing and unequal exchange in the $[4 \mathrm{Fe}-4 \mathrm{~S}]$ cluster}

\section{Single orbital Anderson model}

The direct extrapolation of the simple (single orbital per Fe) Anderson model described in Eq. (4) to the case of [4Fe-4S] cluster takes the form

$$
H=\sum_{i j} J_{i j}\left(S_{i} \cdot S_{j}+S_{i} \cdot s_{j}+S_{j} \cdot s_{i}\right)+\sum_{\sigma=\uparrow, \downarrow} \beta_{i j}\left(c_{i \sigma}^{\dagger} c_{j \sigma}+c_{j \sigma}^{\dagger} c_{i \sigma}\right)
$$

with 6 Heisenberg coupling coefficients $J_{i j}$ and 6 hopping integrals $\beta_{i j}$. We assume that there are two hopping electrons, and that the two electrons cannot be on the same Fe atom due to on-site repulsion.

We solve the above Hamiltonian numerically in the space where the spins $s_{i}$ and $S_{i}=5 / 2$ are antiferromagnetically aligned. The nature of the states changes as the ratio of the exchange coupling coefficients changes. In Supplementary Figure 8 we take $B=2 J^{\prime}$ and $J_{12}, J_{34}=J^{\prime}, J_{13}, J_{14}, J_{23}, J_{24}=J$. For $J / J^{\prime}>1.2$, 


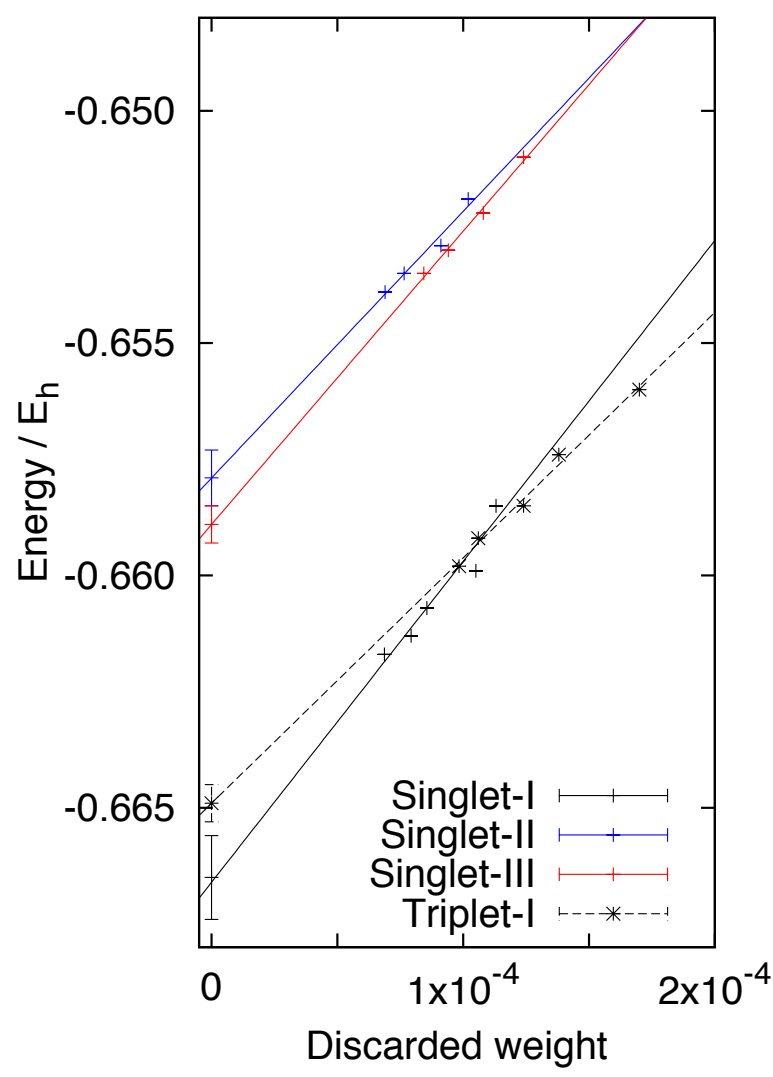

Supplementary Figure 7: DMRG energy (E+8471.0) in $E_{h}$ of the ground-state singlet (Singlet-I) and triplet (Triplet-I) states versus the discarded weight of the $\left[\mathrm{Fe}_{4} \mathrm{~S}_{4}\left(\mathrm{SCH}_{3}\right)_{4}\right]^{-2}$ cluster. In addition, the energies of Singlet-II and Singlet-III correspond to the states where the spin pairing is constrained be non-optimal (see text). The best fit straight lines are extrapolated to zero discarded weight, with the shown error bars, to obtain an estimated FCI energy.

we recover a single pairing picture for the $S_{12}=9 / 2$ dimer states that is assumed in the generalized HDE model of Noodleman et al. [17-21].

The difference in the $J_{i j}$ parameters can be estimated from energy differences between the ground state singlet and triplet states and the difference between the ground state singlet state (Singlet-I) and artificially paired singlet (Singlet-II). The energy difference between the singlet and the triplet state is given by

$$
E(S)-E(T)=J-0.08|B|
$$




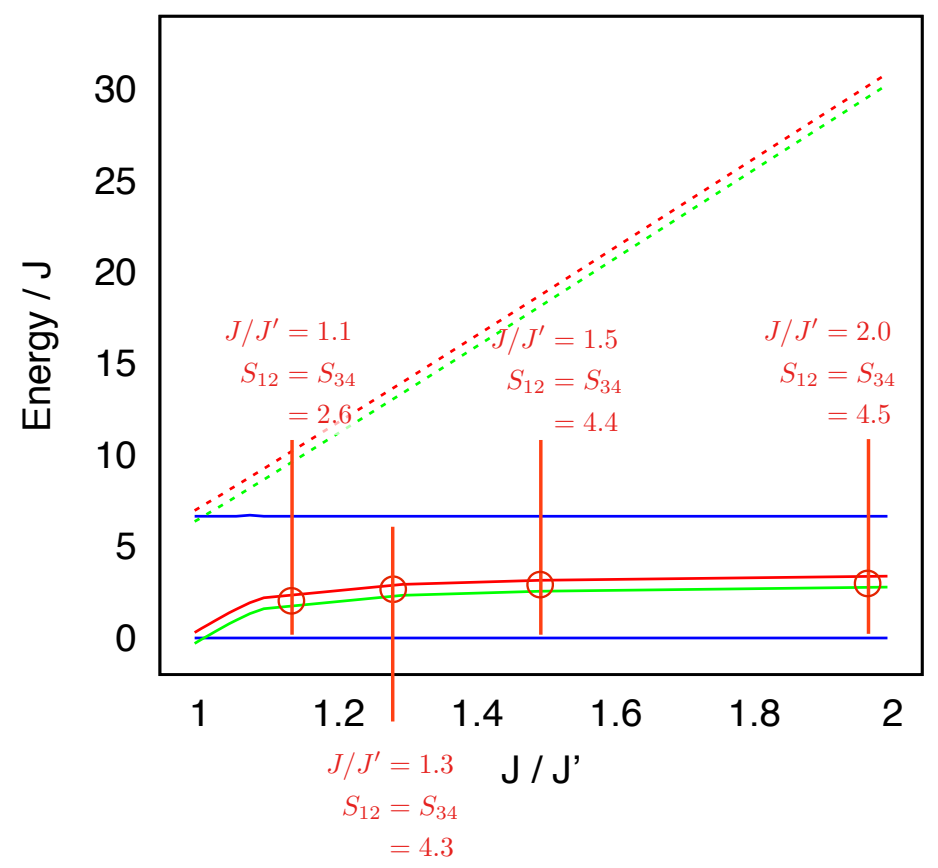

Supplementary Figure 8: Simplified Anderson model energy levels for the [4Fe-4S] cluster, as a function of anisotropic exchange $J^{\prime} \neq J$. As the anisotropy increases, we recover the fixed-pairing picture assumed by the HDE model.

and the energy difference between Singlet-I and Singlet-II states is given by

$$
E\left(S_{I}\right)-E\left(S_{I I}\right)=22.5\left(J-J^{\prime}\right)
$$

In Eq. 9 we assume $B=J$ so that the singlet-triplet gap is $0.92 J$. In Eq. 10 we have assumed that difference in the value of $B$ for different couplings is relatively small. Using the converged DMRG energies we obtain $J=382$ $\mathrm{cm}^{-1}$ and $J-J^{\prime}=84 \mathrm{~cm}^{-1}$, from which we conclude that $J / J^{\prime} \approx 1.28$ and the [4Fe-4S] low-lying states with high effective dimer spin can be described by the single pairing picture, although they appear to lie close to the border of validity of that description. 


\section{Multi-orbital Anderson model}

To check that the above analysis holds in the more complex case of multi-orbital double exchange, we have also analyzed some multi-orbital (per Fe) Anderson models for the [4Fe-4S] cluster. These take the form

$$
H=\sum_{i A B} J_{i A B} s_{i A} \cdot s_{i B}+\sum_{i A B \sigma} \beta_{i A B}\left(c_{i A \sigma}^{\dagger} c_{i B \sigma}+c_{i B \sigma}^{\dagger} c_{i A \sigma}\right)+\sum_{i A \sigma} \Delta_{i A} c_{i A \sigma}^{\dagger} c_{i A \sigma}
$$

where $A$ and $B$ now range over the $4 \mathrm{Fe}$ atoms. The index $i$ ranges over the number of d orbitals on each Fe atom. This would be 5 orbitals in the real cubans, but solving the Hamiltonian for all its levels would be prohibitively expensive. We have therefore considered simpler versions (which illustrate the appropriate trends) where each model Fe atom has respectively only 1 or 2 orbitals. Note that in the 1 orbital case, the maximum spin on each Fe is then only $1 / 2$, and the maximum dimer spin is also $1 / 2$, while in the 2 orbital case, the maximum spin on each Fe is 1 and the maximum dimer spin is $3 / 2$. (The 1 orbital model is related to the Hubbard model on a tetrahedron as discussed in Refs. [22]). In Supplementary Figures 9 and 10 we plot the effective dimer spins of the energy levels as a function of the exchange couping ratio (inequivalent $J$ 's), for $\beta_{i A B}=2 J^{\prime}$ and $\Delta_{i A}=0$ (for all $i, A$ ). We see the same general trends as in the simple Anderson model above. Note that $J_{c}$ shifts to lower values as the number of orbitals on each Fe increases. 


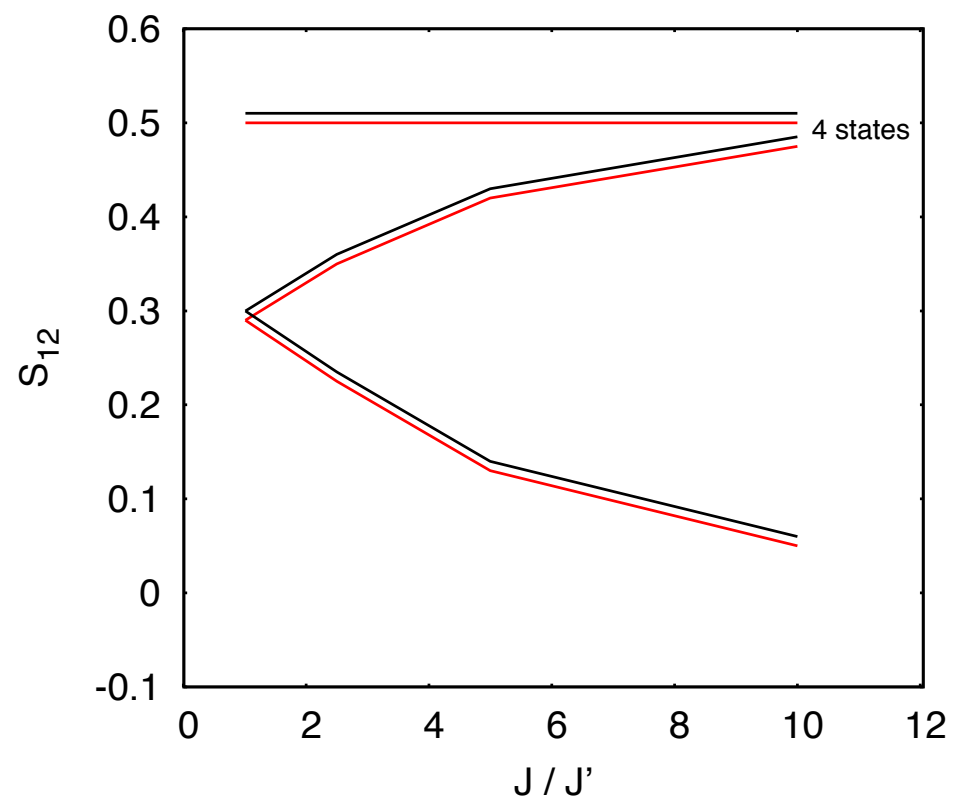

Supplementary Figure 9: Effective dimer spins of the energy levels as a function of exchange coupling ratio for the [4Fe4S] multi-orbital Anderson model with 1 orbital per model Fe atom. Note that since the "Fe" atom has only one orbital, the maximum spin per atom is $1 / 2$, and the maximum dimer spin is $1 / 2$.

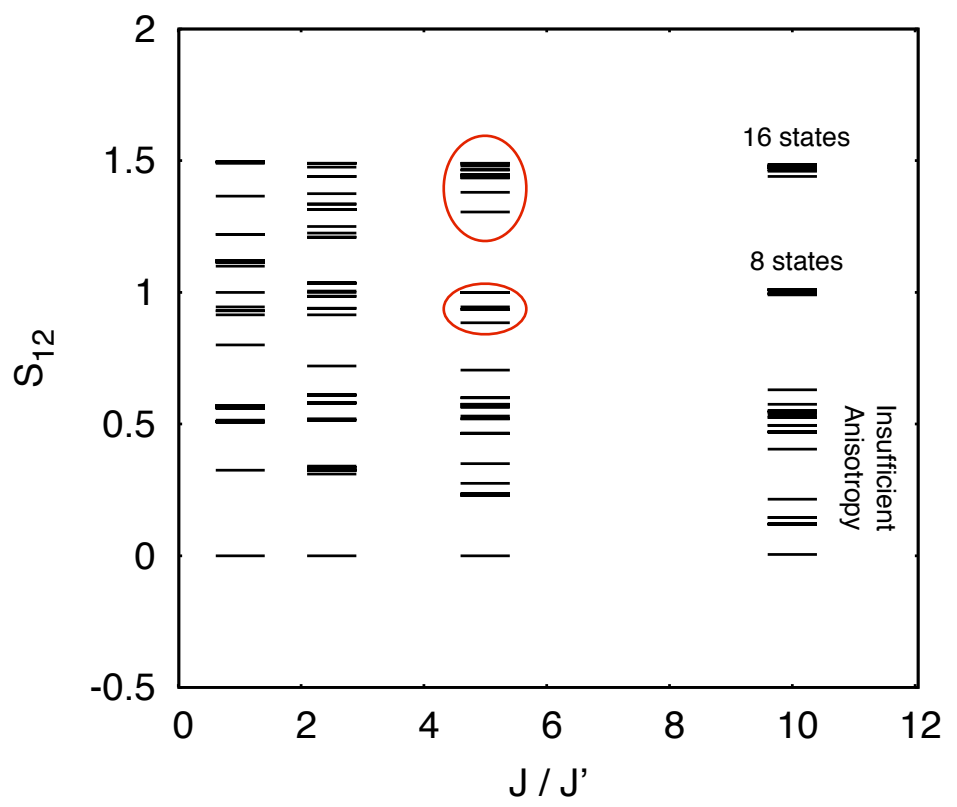

Supplementary Figure 10: Effective dimer spins of the energy levels as a function of exchange coupling ratio for the [4Fe-4S] multi-orbital Anderson model with 2 orbitals per Fe. Note that since the "Fe" atom has only two orbitals, the maximum spin per atom is 1 , and the maximum dimer spin is $3 / 2$. 
[1] J. J. Mayerle, S. E. Denmark, B. V. DePamphilis, J. A. Ibers, R. H. Holm, Journal of the American Chemical Society 97, $1032(1975)$.

[2] A. Schafer, H. Horn, R. Ahlrichs, J. Chem. Phys. 97, 2571 (1992).

[3] F. Neese, Wiley Interdisciplinary Reviews: Computational Molecular Science 2, 73 (2012).

[4] L. Bytautas, J. Ivanic, K. Ruedenberg, The Journal of Chemical Physics 119, 8217 (2003).

[5] J. Pipek, P. G. Mezey, The Journal of Chemical Physics 90, 4916 (1989).

[6] S. Sharma, G. K.-L. Chan, The Journal of Chemical Physics 136, 124121 (2012).

[7] K. Andersson, B. O. Roos, Chemical Physics Letters 191, 507 (1992).

[8] G. K.-L. Chan, M. Head-Gordon, The Journal of Chemical Physics 118, 8551 (2003).

[9] E. Ramos-Cordoba, E. Matito, I. Mayer, P. Salvador, Journal of Chemical Theory and Computation 8, 1270 (2012).

[10] K. Yamaguchi, T. Fueno, Chemical Physics 19, 35 (1977).

[11] K. Yamaguchi, Chemical Physics 29, 117 (1978).

[12] P. W. Anderson, H. Hasegawa, Physical Review 100, 675 (1955).

[13] L. Noodleman, E. J. Baerends, Journal of the American Chemical Society 106, 2316 (1984).

[14] M. Shoji, et al., International Journal of Quantum Chemistry 107, 609 (2007).

[15] J. Rawlings, O. Siiman, H. B. Gray, Proceedings of the National Academy of Sciences 71, 125 (1974).

[16] F. Weigend, R. Ahlrichs, Phys. Chem. Chem. Phys. 7, 3297 (2005).

[17] L. Noodleman, C. Y. Peng, D. A. Case, J. M. Mouesca, Coordination Chemistry Reviews 144, 199 (1995).

[18] K. Yamaguchi, Y. Yoshioka, T. Takatsuka, T. Fueno, Theoretica chimica acta 48, 185 (1978).

[19] K. Yamaguchi, T. Fueno, N. Ueyama, N. Akira, O. Masaaki, Chemical Physics Letters 164, 210 (1989).

[20] Y. Kitagawa, et al., International Journal of Quantum Chemistry 108, 2881 (2008).

[21] M. Nishino, et al., Bulletin of the Chemical Society of Japan 71, 99 (1998).

[22] L. Falicov, R. Victora, Physical Review B 30, 1695 (1984). 
Supplementary Table 6: Unrelaxed geometry $\left[\mathrm{Fe}_{2} \mathrm{~S}_{2}\left(\mathrm{SCH}_{3}\right)_{4}\right]^{3-}$ energy $\left(\mathrm{cm}^{-1}\right)$, local population, total spin and spincorrelation functions for the lowest ten levels in each dimer total spin state, using active space (2).

\begin{tabular}{|c|c|c|c|c|c|c|c|c|c|c|}
\hline State & 1 & 2 & 3 & 4 & 5 & 6 & 7 & 8 & 9 & 10 \\
\hline \multicolumn{11}{|l|}{$S=1 / 2$} \\
\hline$E$ & 0 & 325 & 1132 & 2642 & 4264 & 4989 & 4905 & 5313 & 6448 & 7049 \\
\hline$\left\langle N_{1}\right\rangle$ & 6.23 & 6.25 & 6.24 & 6.23 & 6.22 & 6.21 & 6.22 & 6.21 & 6.21 & 6.21 \\
\hline$\left\langle N_{2}\right\rangle$ & 6.24 & 6.23 & 6.24 & 6.23 & 6.22 & 6.22 & 6.21 & 6.21 & 6.21 & 6.21 \\
\hline$\left\langle S_{1}\right\rangle$ & 5.39 & 5.31 & 5.34 & 5.35 & 5.39 & 5.40 & 5.36 & 5.43 & 5.40 & 5.42 \\
\hline$\left\langle S_{2}\right\rangle$ & 5.32 & 5.36 & 5.34 & 5.35 & 5.36 & 5.37 & 5.43 & 5.42 & 5.41 & 5.42 \\
\hline$\left\langle S_{1} \cdot S_{2}\right\rangle$ & -4.74 & -4.77 & -4.81 & -4.62 & -4.84 & -4.72 & -4.83 & -4.89 & -4.78 & -4.77 \\
\hline \multicolumn{11}{|l|}{$S=3 / 2$} \\
\hline$E$ & 136 & 527 & 1710 & 4264 & 4451 & 5030 & 5131 & 6073 & 7870 & 8581 \\
\hline$\left\langle N_{1}\right\rangle$ & 6.22 & 6.24 & 6.24 & 6.22 & 6.21 & 6.21 & 6.21 & 6.22 & 6.21 & 6.20 \\
\hline$\left\langle N_{2}\right\rangle$ & 6.23 & 6.24 & 6.23 & 6.22 & 6.22 & 6.21 & 6.21 & 6.21 & 6.21 & 6.20 \\
\hline$\left\langle S_{1}\right\rangle$ & 5.42 & 5.35 & 5.34 & 5.37 & 5.40 & 5.42 & 5.43 & 5.38 & 5.42 & 5.43 \\
\hline$\left\langle S_{2}\right\rangle$ & 5.39 & 5.36 & 5.36 & 5.37 & 5.40 & 5.42 & 5.43 & 5.41 & 5.42 & 5.43 \\
\hline$\left\langle S_{1} \cdot S_{2}\right\rangle$ & -3.66 & -3.77 & -3.64 & -3.38 & -3.68 & -3.79 & -3.78 & -3.47 & -3.61 & -3.59 \\
\hline \multicolumn{11}{|l|}{$S=5 / 2$} \\
\hline$E$ & 336 & 643 & 2871 & 4668 & 5300 & 5678 & 6248 & 7580 & 9459 & 10260 \\
\hline$\left\langle N_{1}\right\rangle$ & 6.21 & 6.23 & 6.23 & 6.20 & 6.21 & 6.20 & 6.22 & 6.21 & 6.20 & 6.20 \\
\hline$\left\langle N_{2}\right\rangle$ & 6.22 & 6.24 & 6.23 & 6.21 & 6.21 & 6.21 & 6.22 & 6.20 & 6.20 & 6.20 \\
\hline$\left\langle S_{1}\right\rangle$ & 5.50 & 5.38 & 5.37 & 5.45 & 5.44 & 5.46 & 5.38 & 5.41 & 5.44 & 5.43 \\
\hline$\left\langle S_{2}\right\rangle$ & 5.45 & 5.37 & 5.38 & 5.43 & 5.44 & 5.44 & 5.39 & 5.45 & 5.45 & 5.44 \\
\hline$\left\langle S_{1} \cdot S_{2}\right\rangle$ & -1.89 & -1.96 & -1.77 & -1.81 & -1.91 & -1.95 & -1.33 & -1.51 & -1.68 & -1.67 \\
\hline \multicolumn{11}{|l|}{$S=7 / 2$} \\
\hline$E$ & 669 & 1330 & 4675 & 5441 & 6358 & 7049 & 8989 & 9589 & 9913 & 10115 \\
\hline$\left\langle N_{1}\right\rangle$ & 6.19 & 6.22 & 6.22 & 6.19 & 6.19 & 6.20 & 6.20 & 6.25 & 6.22 & 6.28 \\
\hline$\left\langle N_{2}\right\rangle$ & 6.20 & 6.23 & 6.22 & 6.19 & 6.21 & 6.19 & 6.21 & 6.27 & 6.24 & 6.30 \\
\hline$\left\langle S_{1}\right\rangle$ & 5.56 & 5.43 & 5.43 & 5.50 & 5.51 & 5.50 & 5.45 & 5.02 & 5.29 & 4.76 \\
\hline$\left\langle S_{2}\right\rangle$ & 5.55 & 5.42 & 5.44 & 5.51 & 5.46 & 5.54 & 5.40 & 5.00 & 5.25 & 4.76 \\
\hline$\left\langle S_{1} \cdot S_{2}\right\rangle$ & 0.55 & 0.60 & 0.78 & 0.78 & 0.67 & 0.61 & 1.38 & 2.16 & 1.72 & 2.49 \\
\hline \multicolumn{11}{|l|}{$S=9 / 2$} \\
\hline$E$ & 1071 & 2943 & 7357 & 7403 & 8496 & 9209 & 13263 & 13699 & 14803 & 15073 \\
\hline$\left\langle N_{1}\right\rangle$ & 6.18 & 6.21 & 6.16 & 6.20 & 6.19 & 6.17 & 6.17 & 6.17 & 6.17 & 6.22 \\
\hline$\left\langle N_{2}\right\rangle$ & 6.19 & 6.21 & 6.16 & 6.20 & 6.18 & 6.17 & 6.17 & 6.17 & 6.17 & 6.22 \\
\hline$\left\langle S_{1}\right\rangle$ & 5.63 & 5.57 & 5.68 & 5.59 & 5.60 & 5.64 & 5.65 & 5.65 & 5.64 & 5.53 \\
\hline$\left\langle S_{2}\right\rangle$ & 5.62 & 5.56 & 5.67 & 5.58 & 5.62 & 5.65 & 5.66 & 5.65 & 5.64 & 5.53 \\
\hline$\left\langle S_{1} \cdot S_{2}\right\rangle$ & 3.62 & 3.58 & 3.67 & 3.60 & 3.62 & 3.65 & 3.66 & 3.65 & 3.65 & 3.56 \\
\hline
\end{tabular}


Supplementary Table 7: Unrelaxed geometry $\left[\mathrm{Fe}_{2} \mathrm{~S}_{2}\left(\mathrm{SCH}_{3}\right)_{4}\right]^{3-}$ energy $\left(\mathrm{cm}^{-1}\right)$, local population, total spin and spincorrelation functions for the lowest ten levels in each dimer total spin state, using active space (2). The orbitals taken to

\begin{tabular}{|c|c|c|c|c|c|c|c|c|c|c|}
\hline Sta & 1 & 2 & 3 & 4 & 5 & 6 & 7 & 8 & 9 & 10 \\
\hline \multicolumn{11}{|l|}{$S=1 / 2$} \\
\hline$E$ & 0 & & 2 & 2 & 264 & 9 & 4905 & 3 & 8 & 049 \\
\hline$\left\langle N_{1}\right\rangle$ & 5.48 & & 9 & & 9 & & 2 & 49 & & 49 \\
\hline$\left\langle N_{2}\right\rangle$ & 52 & & 1 & 51 & 1 & 52 & 5.48 & 51 & 50 & 5.51 \\
\hline$\left\langle S_{1}\right\rangle$ & 87 & 5.63 & 77 & 4 & 5.84 & 78 & 2 & .84 & 5.74 & 5.76 \\
\hline$\left\langle S_{2}\right\rangle$ & 51 & & & 5.68 & 63 & 9 & 5 & 3 & 9 & 5.78 \\
\hline$\left\langle S_{1} \cdot S_{2}\right\rangle$ & -5.37 & -5.36 & -5.39 & -5.28 & .36 & -5.31 & -5.41 & -5.46 & -5.39 & -5.40 \\
\hline \multicolumn{11}{|l|}{$S=3 / 2$} \\
\hline$E$ & 6 & 27 & 10 & 264 & 4451 & 5030 & 5131 & 973 & 7870 & 8581 \\
\hline$\left\langle N_{1}\right\rangle$ & 15.49 & & 15.50 & 15.49 & 15.50 & 15.49 & 15.49 & 15.50 & 15.50 & 15.49 \\
\hline$\left\langle N_{2}\right\rangle$ & 15.51 & 15.50 & 15.50 & 15.51 & 15.50 & 15.51 & 15.51 & 15.50 & 15.50 & 15.51 \\
\hline$\left\langle S_{1}\right\rangle$ & 6.00 & 5.95 & 5.85 & 5.76 & 5.93 & 6.00 & 5.99 & 5.74 & 5.89 & 5.90 \\
\hline$\left\langle S_{2}\right\rangle$ & 5.89 & 5.98 & 92 & 5.78 & 5.86 & 5.95 & 5.98 & 5.84 & 5.91 & 5.88 \\
\hline$\left\langle S_{1} \cdot S_{2}\right\rangle$ & -4.07 & -4.09 & -4.01 & -3.90 & -4.02 & -4.10 & -4.11 & -3.91 & -4.03 & -4.01 \\
\hline \multicolumn{11}{|l|}{$S=5 / 2$} \\
\hline$E$ & 33 & & 2871 & 4668 & 5300 & 5678 & 6 & 7580 & 9459 & 10260 \\
\hline$\left\langle N_{1}\right\rangle$ & 15.49 & 49 & 15.50 & 15.50 & 15.49 & 15.49 & 15.49 & 15.50 & 15.50 & 15.49 \\
\hline$\left\langle N_{2}\right\rangle$ & 15.51 & 15.51 & 15.50 & 15.50 & 15.51 & 15.51 & 15.51 & 15.50 & 15.50 & 15.51 \\
\hline$\left\langle S_{1}\right\rangle$ & 6.35 & 6.27 & 6.13 & 6.24 & 6.24 & 6.32 & 5.91 & 5.95 & 6.11 & 6.12 \\
\hline$\left\langle S_{2}\right\rangle$ & 6.22 & 6. & 17 & 6.1 & 6.26 & 6.16 & 5 & 6.10 & 6.16 & 6.13 \\
\hline$\left\langle S_{1} \cdot S_{2}\right\rangle$ & -1.91 & 89 & -1.78 & -1.84 & -1.87 & -1.87 & -1.55 & -1.65 & -1.76 & -1.75 \\
\hline \multicolumn{11}{|l|}{$S=7 / 2$} \\
\hline$E$ & & & 5 & 54 & 5 & 70 & 8 & 9 & 9913 & 10115 \\
\hline$\left\langle N_{1}\right\rangle$ & 15.49 & 9 & 15.50 & 15.50 & 15.48 & 15.50 & 15.48 & 15.46 & 15.46 & 15.54 \\
\hline$\left\langle N_{2}\right\rangle$ & 15.51 & 15.51 & 15.50 & 15.50 & 15.52 & 15.50 & 15.52 & 15.54 & 15.54 & 15.46 \\
\hline$\left\langle S_{1}\right\rangle$ & & & & 6.56 & 6.70 & 6.62 & 6.31 & 5.59 & 5.98 & 5.46 \\
\hline$\left\langle S_{2}\right\rangle$ & & & & 6.67 & 6.62 & 6.76 & 6.19 & 5.65 & 5.97 & 5.21 \\
\hline$\left\langle S_{1} \cdot S_{2}\right\rangle$ & 10 & 1.19 & 1.30 & 1.26 & 1.22 & 1.19 & 1.63 & 2.26 & 1.90 & 2.54 \\
\hline \multicolumn{11}{|l|}{$S=9 / 2$} \\
\hline$\pi$ & & & ונואנו & 7403 & 8496 & 9209 & 13263 & 13699 & 14803 & 15073 \\
\hline$\left\langle N_{1}\right\rangle$ & 15.49 & 15.49 & 15.50 & 15.49 & 15.50 & 15.49 & 15.48 & 15.51 & 15.49 & 15.50 \\
\hline$\left\langle N_{2}\right\rangle$ & 15.51 & 15.51 & 15.50 & 15.51 & 15.50 & 15.51 & 15.52 & 15.49 & 15.51 & 15.50 \\
\hline$\left\langle S_{1}\right\rangle$ & 7.44 & 7.43 & 7.41 & 7.45 & 7.42 & 7.44 & 7.46 & 7.39 & 7.44 & 7.42 \\
\hline$\left\langle S_{2}\right\rangle$ & 7.40 & 7.40 & 7.40 & 7.38 & 7.41 & 7.39 & 7.37 & 7.44 & 7.38 & 7.40 \\
\hline$\left.S_{1} \cdot S_{2}\right\rangle$ & 4.95 & 4.96 & 4.97 & 4.96 & 4.96 & 4.96 & 4.96 & 4.96 & 4.96 & 4.96 \\
\hline
\end{tabular}


Supplementary Table 8: Relaxed geometry $\left[\mathrm{Fe}_{2} \mathrm{~S}_{2}\left(\mathrm{SCH}_{3}\right)_{4}\right]^{3-}$ energy $\left(\mathrm{cm}^{-1}\right)$, local population, total spin and spin-correlation functions for the lowest ten levels in each dimer total spin state, using active space (2).

\begin{tabular}{|c|c|c|c|c|c|c|c|c|c|c|}
\hline State & 1 & 2 & 3 & 4 & 5 & 6 & 7 & 8 & 9 & 10 \\
\hline \multicolumn{11}{|l|}{$S=1 / 2$} \\
\hline$E$ & 0 & 1218 & 2079 & 3790 & 4070 & 4314 & 4885 & 5731 & 6975 & 7644 \\
\hline$\left\langle N_{1}\right\rangle$ & 6.26 & 6.24 & 6.16 & 6.18 & 6.21 & 6.21 & 6.20 & 6.17 & 6.17 & 6.17 \\
\hline$\left\langle N_{2}\right\rangle$ & 6.14 & 6.16 & 6.28 & 6.25 & 6.16 & 6.16 & 6.16 & 6.23 & 6.22 & 6.22 \\
\hline$\left\langle S_{1}\right\rangle$ & 5.32 & 5.37 & 5.67 & 5.63 & 5.45 & 5.42 & 5.48 & 5.62 & 5.65 & 5.64 \\
\hline$\left\langle S_{2}\right\rangle$ & 5.73 & 5.67 & 5.18 & 5.26 & 5.68 & 5.67 & 5.65 & 5.33 & 5.34 & 5.31 \\
\hline$\left\langle S_{1} \cdot S_{2}\right\rangle$ & -4.96 & -5.01 & -4.85 & -4.78 & -5.03 & -5.04 & -5.02 & -4.86 & -4.90 & -4.89 \\
\hline \multicolumn{11}{|l|}{$S=3 / 2$} \\
\hline$E$ & 266 & 1211 & 2374 & 4197 & 4493 & 4694 & 4925 & 6460 & 7693 & 8342 \\
\hline$\left\langle N_{1}\right\rangle$ & 6.24 & 6.23 & 6.17 & 6.20 & 6.19 & 6.18 & 6.19 & 6.17 & 6.17 & 6.24 \\
\hline$\left\langle N_{2}\right\rangle$ & 6.15 & 6.17 & 6.26 & 6.18 & 6.19 & 6.21 & 6.16 & 6.22 & 6.21 & 6.28 \\
\hline$\left\langle S_{1}\right\rangle$ & 5.37 & 5.42 & 5.64 & 5.50 & 5.53 & 5.57 & 5.49 & 5.61 & 5.63 & 5.37 \\
\hline$\left\langle S_{2}\right\rangle$ & 5.72 & 5.63 & 5.28 & 5.61 & 5.51 & 5.44 & 5.67 & 5.39 & 5.39 & 4.93 \\
\hline$\left\langle S_{1} \cdot S_{2}\right\rangle$ & -3.86 & -3.97 & -3.75 & -3.90 & -3.74 & -3.67 & -3.89 & -3.61 & -3.72 & -2.71 \\
\hline \multicolumn{11}{|l|}{$S=5 / 2$} \\
\hline$E$ & 623 & 1323 & 2848 & 4619 & 4913 & 5200 & 5943 & 7536 & 8374 & 8715 \\
\hline$\left\langle N_{1}\right\rangle$ & 6.23 & 6.22 & 6.18 & 6.18 & 6.18 & 6.19 & 6.18 & 6.17 & 6.24 & 6.18 \\
\hline$\left\langle N_{2}\right\rangle$ & 6.15 & 6.18 & 6.22 & 6.20 & 6.17 & 6.16 & 6.23 & 6.21 & 6.28 & 6.21 \\
\hline$\left\langle S_{1}\right\rangle$ & 5.44 & 5.47 & 5.59 & 5.57 & 5.55 & 5.52 & 5.60 & 5.62 & 5.34 & 5.61 \\
\hline$\left\langle S_{2}\right\rangle$ & 5.70 & 5.62 & 5.44 & 5.52 & 5.61 & 5.67 & 5.38 & 5.43 & 4.98 & 5.41 \\
\hline$\left\langle S_{1} \cdot S_{2}\right\rangle$ & -2.03 & -2.16 & -1.96 & -1.98 & -2.04 & -2.03 & -1.43 & -1.59 & -0.24 & -1.76 \\
\hline \multicolumn{11}{|l|}{$S=7 / 2$} \\
\hline$E$ & 786 & 1405 & 3375 & 5173 & 5368 & 5696 & 7528 & 8254 & 8863 & 9149 \\
\hline$\left\langle N_{1}\right\rangle$ & 6.21 & 6.21 & 6.20 & 6.17 & 6.17 & 6.18 & 6.17 & 6.24 & 6.17 & 6.24 \\
\hline$\left\langle N_{2}\right\rangle$ & 6.15 & 6.18 & 6.18 & 6.17 & 6.21 & 6.16 & 6.22 & 6.27 & 6.20 & 6.27 \\
\hline$\left\langle S_{1}\right\rangle$ & 5.52 & 5.51 & 5.55 & 5.60 & 5.63 & 5.57 & 5.61 & 5.33 & 5.62 & 5.33 \\
\hline$\left\langle S_{2}\right\rangle$ & 5.70 & 5.62 & 5.61 & 5.62 & 5.52 & 5.67 & 5.42 & 5.03 & 5.47 & 5.06 \\
\hline$\left\langle S_{1} \cdot S_{2}\right\rangle$ & 0.50 & 0.39 & 0.53 & 0.60 & 0.60 & 0.52 & 1.33 & 3.07 & 1.18 & 2.98 \\
\hline \multicolumn{11}{|l|}{$S=9 / 2$} \\
\hline$E$ & 984 & 1839 & 4254 & 5941 & 6458 & 6873 & 10136 & 11196 & 11377 & 12487 \\
\hline$\left\langle N_{1}\right\rangle$ & 6.19 & 6.20 & 6.20 & 6.16 & 6.17 & 6.15 & 6.15 & 6.15 & 6.18 & 6.16 \\
\hline$\left\langle N_{2}\right\rangle$ & 6.16 & 6.18 & 6.15 & 6.14 & 6.17 & 6.21 & 6.20 & 6.19 & 6.21 & 6.19 \\
\hline$\left\langle S_{1}\right\rangle$ & 5.60 & 5.57 & 5.56 & 5.68 & 5.66 & 5.71 & 5.70 & 5.70 & 5.64 & 5.69 \\
\hline$\left\langle S_{2}\right\rangle$ & 5.72 & 5.66 & 5.73 & 5.74 & 5.67 & 5.55 & 5.58 & 5.60 & 5.56 & 5.59 \\
\hline$\left\langle S_{1} \cdot S_{2}\right\rangle$ & 3.66 & 3.63 & 3.66 & 3.71 & 3.68 & 3.65 & 3.66 & 3.67 & 3.63 & 3.66 \\
\hline
\end{tabular}


Supplementary Table 9: Relaxed geometry $\left[\mathrm{Fe}_{2} \mathrm{~S}_{2}\left(\mathrm{SCH}_{3}\right)_{4}\right]^{3-}$ energy $\left(\mathrm{cm}^{-1}\right)$, local population, total spin and spin-correlation functions for the lowest ten levels in each dimer total spin state, using active space (2).

\begin{tabular}{|c|c|c|c|c|c|c|c|c|c|c|}
\hline State & 1 & 2 & 3 & 4 & 5 & 6 & 7 & 8 & 9 & 10 \\
\hline \multicolumn{11}{|l|}{$S=1 / 2$} \\
\hline$E$ & 0 & 8 & 2079 & 0 & 4070 & 4314 & 5 & 5731 & 975 & 7644 \\
\hline$\left\langle N_{1}\right\rangle$ & 15.62 & 59 & 15.37 & 39 & 1 & 61 & & 5.41 & 37 & 15.38 \\
\hline$\left\langle N_{2}\right\rangle$ & 38 & 15.41 & 15.63 & 15.61 & 15.39 & 15.39 & 15.42 & 15.59 & 15.63 & 15.62 \\
\hline$\left\langle S_{1}\right\rangle$ & 05 & 5.20 & 7.31 & 7.11 & 5.24 & 5.26 & 5.30 & 6.89 & 7.28 & 7.23 \\
\hline$\left\langle S_{2}\right\rangle$ & 7.42 & 7.25 & 5.04 & 5.13 & 7.30 & 7.27 & 7.02 & 5.31 & 5.11 & 5.02 \\
\hline$\left\langle S_{1} \cdot S_{2}\right\rangle$ & -5.86 & -5.85 & -5.80 & -5.74 & -5.89 & -5.89 & -5.79 & -5.72 & 32 & -5.75 \\
\hline \multicolumn{11}{|l|}{$S=3 / 2$} \\
\hline$E$ & 6 & 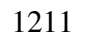 & 74 & 4 & 493 & 4694 & 49 & 64 & 76 & 8342 \\
\hline$\left\langle N_{1}\right\rangle$ & 15.61 & 15.56 & 15.40 & 15.57 & 1 & 15.46 & $1 J$ & 15.45 & 15.40 & 15.30 \\
\hline$\left\langle N_{2}\right\rangle$ & 15.39 & 15.44 & 15.60 & 15.43 & 47 & 15.54 & 15.42 & 15.57 & 15.60 & 15.70 \\
\hline$\left\langle S_{1}\right\rangle$ & Q & 5.70 & .11 & 5.79 & 5.95 & 6.57 & 5 & 0 & 7.09 & 6.21 \\
\hline$\left\langle S_{2}\right\rangle$ & 38 & 7.10 & 51 & 6.96 & 6.43 & 6.05 & 7.07 & 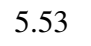 & 5.47 & 5.11 \\
\hline$\left\langle S_{1} \cdot S_{2}\right\rangle$ & -4.51 & -4.53 & -4.43 & -4.50 & -4.32 & -4.43 & -4.44 & & 1 & -3.78 \\
\hline \multicolumn{11}{|l|}{$S=5 / 2$} \\
\hline$E$ & & 32 & 2848 & 46 & & 5200 & 59 & & + & 8715 \\
\hline$\left\langle N_{1}\right\rangle$ & 15.60 & 15.55 & 15.45 & 15.51 & 15.54 & 15.59 & 15.42 & 15.43 & 15 & 15.41 \\
\hline$\left\langle N_{2}\right\rangle$ & 15.40 & 15.45 & 15.55 & 15.49 & 15.46 & 15.41 & 15.58 & 15.57 & 15.71 & 15.59 \\
\hline$\left\langle S_{1}\right\rangle$ & 5.90 & 6.19 & 6.83 & 6.45 & 6.15 & 5.85 & 6.72 & 6.8 & 5.55 & 7.01 \\
\hline$\left\langle S_{2}\right\rangle$ & 7.33 & 7.10 & 6.25 & 6.65 & 6.91 & 7.19 & 5.79 & 5.71 & 5.27 & 5.83 \\
\hline$\left\langle S_{1} \cdot S_{2}\right\rangle$ & -2.24 & -2.27 & -2.16 & -2.18 & -2.16 & -2.15 & -1.88 & -1.92 & -1.03 & -2.05 \\
\hline \multicolumn{11}{|l|}{$S=7 / 2$} \\
\hline$E$ & 6 & 1405 & 3375 & 51 & 8 & 56 & 75 & & 3 & 9149 \\
\hline$\left\langle N_{1}\right\rangle$ & 15.58 & 15.54 & 15.51 & 15.53 & 15.48 & 15.57 & 15.43 & 15 & 15.42 & 15.39 \\
\hline$\left\langle N_{2}\right\rangle$ & 15.42 & 15.46 & 15.49 & 15.47 & 15.52 & 15.43 & 15.57 & 15.72 & 15.58 & 15.61 \\
\hline$\left\langle S_{1}\right\rangle$ & 6.53 & 6.69 & 6.76 & 6.68 & 6.92 & 6.50 & 6.66 & 5.01 & 7.10 & 5.29 \\
\hline$\left\langle S_{2}\right\rangle$ & 37 & 7.23 & 7.00 & 7.06 & 6.69 & 7.26 & 6. & 5.46 & 5.90 & 5.23 \\
\hline$\left\langle S_{1} \cdot S_{2}\right\rangle$ & 92 & 0.92 & 0.99 & 1.00 & 1.07 & 1.00 & 1.46 & 2.64 & 1.38 & 2.61 \\
\hline \multicolumn{11}{|l|}{$S=9 / 2$} \\
\hline$E$ & & 9 & 4254 & 59 & 6 & 68 & 10 & & & 12487 \\
\hline$\left\langle N_{1}\right\rangle$ & 15.56 & 15.54 & 15.57 & 15.55 & 15.54 & 15.42 & 15.48 & 15.29 & 15.37 & 15.44 \\
\hline$\left\langle N_{2}\right\rangle$ & 15.44 & 15.46 & 15.43 & 15.45 & 15.46 & 15.58 & 15.52 & 15.71 & 15.63 & 15.56 \\
\hline$\left\langle S_{1}\right\rangle$ & 7.23 & 7.28 & 7.20 & 7.25 & 7.27 & 7.60 & 7.45 & 7.95 & 7.74 & 7.56 \\
\hline & 7.55 & 7.50 & 7.57 & 7.53 & 7.51 & 7.18 & 7.33 & 6.83 & 7.03 & 7.21 \\
\hline$\left\langle S_{1} \cdot S_{2}\right\rangle$ & 4.98 & 4.99 & 4.99 & 4.98 & 4.99 & 4.98 & 4.99 & 4.98 & 4.99 & 4.99 \\
\hline
\end{tabular}


Supplementary Table 10: The best-fit parameters $\left(\mathrm{cm}^{-1}\right)$ of the extended Anderson's double exchange Hamiltonian given in Equation 6 used to fit the low-lying energy levels of the $\left[\mathrm{Fe}_{2} \mathrm{~S}_{2}\left(\mathrm{SCH}_{3}\right)_{4}\right]^{3-}$ dimer. Note that $J_{i}$ and $\beta_{i}$ do not correspond to the exchange and double exchange parameters in the standard HDE model and cannot be directly compared. Standard deviations (as estimated from the covariance matrix of the fit) given in brackets.

\begin{tabular}{|c|c|c|}
\hline$J_{i}$ & $\beta_{i}$ & $\Delta_{i}$ \\
\hline $12656( \pm 513)$ & $3512( \pm 280)$ & \\
\hline $22743( \pm 682)$ & $9679( \pm 294)$ & $1536( \pm 271)$ \\
\hline $32151( \pm 518)$ & $4653( \pm$ & $4433( \pm 196)$ \\
\hline $41756( \pm 675)$ & $8472( \pm 294)$ & $6167( \pm 268)$ \\
\hline $5395( \pm 695)$ & $6562( \pm 296)$ & $6167( \pm 284)$ \\
\hline
\end{tabular}

Supplementary Table 11: Coordinates (in $\AA$ ) of the $\left[\mathrm{Fe}_{4} \mathrm{~S}_{4}\left(\mathrm{SCH}_{3}\right)_{4}\right]^{2-}$ model complex.

\begin{tabular}{ccccc}
\hline \hline & & $\mathrm{x}$ & $\mathrm{y}$ & $\mathrm{z}$ \\
\hline 1 & $\mathrm{~S}$ & 0.04 & -1.78 & -1.29 \\
2 & $\mathrm{~S}$ & -0.04 & 1.78 & -1.29 \\
3 & $\mathrm{~S}$ & 1.78 & -0.04 & 1.29 \\
4 & $\mathrm{~S}$ & -1.78 & 0.04 & 1.29 \\
5 & $\mathrm{Fe}$ & 0.05 & -1.37 & 1.01 \\
6 & $\mathrm{Fe}$ & -1.38 & 0.05 & -1.00 \\
7 & $\mathrm{Fe}$ & -0.05 & 1.38 & 1.00 \\
8 & $\mathrm{Fe}$ & 1.37 & -0.05 & -1.01 \\
9 & $\mathrm{~S}$ & 0.24 & 3.30 & 2.14 \\
10 & $\mathrm{~S}$ & -0.24 & -3.29 & 2.14 \\
11 & $\mathrm{~S}$ & -3.29 & -0.24 & -2.14 \\
12 & $\mathrm{~S}$ & 3.29 & 0.24 & -2.14 \\
13 & $\mathrm{C}$ & -3.80 & -1.84 & -1.38 \\
14 & $\mathrm{H}$ & -3.91 & -1.71 & -0.29 \\
15 & $\mathrm{H}$ & -4.76 & -2.17 & -1.81 \\
16 & $\mathrm{H}$ & -3.03 & -2.60 & -1.56 \\
17 & $\mathrm{C}$ & 3.80 & 1.83 & -1.38 \\
18 & $\mathrm{H}$ & 3.91 & 1.71 & -0.29 \\
19 & $\mathrm{H}$ & 4.76 & 2.16 & -1.81 \\
20 & $\mathrm{H}$ & 3.03 & 2.59 & -1.55 \\
21 & $\mathrm{C}$ & -1.83 & -3.80 & 1.38 \\
22 & $\mathrm{H}$ & -2.16 & -4.76 & 1.81 \\
23 & $\mathrm{H}$ & -2.59 & -3.03 & 1.55 \\
24 & $\mathrm{H}$ & -1.70 & -3.91 & 0.29 \\
25 & $\mathrm{C}$ & 1.84 & 3.80 & 1.38 \\
26 & $\mathrm{H}$ & 2.17 & 4.76 & 1.81 \\
27 & $\mathrm{H}$ & 2.60 & 3.03 & 1.56 \\
28 & $\mathrm{H}$ & 1.71 & 3.91 & 0.29 \\
\hline \hline & & & &
\end{tabular}


Supplementary Table 12: Local population and spin correlation function for the four Fe atoms in the calculated DMRG states of the $\left[\mathrm{Fe}_{4} \mathrm{~S}_{4}\left(\mathrm{SCH}_{3}\right)_{4}\right]^{-2}$ cluster.

\begin{tabular}{cccccc}
\hline \hline \multirow{2}{*}{ Fe atom $\left\langle N_{i}\right\rangle$} & \multicolumn{4}{c}{$\left\langle S_{i} \cdot S_{j}\right\rangle$} \\
& & $\mathrm{Fe} 1$ & $\mathrm{Fe} 2$ & $\mathrm{Fe} 3$ & $\mathrm{Fe} 4$ \\
\hline Singlet-I & & & & \\
\hline Fe1 & 6.27 & 5.27 & 3.24 & -4.05 & -4.05 \\
Fe2 & 6.27 & 3.24 & 5.26 & -4.05 & -4.04 \\
Fe3 & 6.27 & -4.05 & -4.05 & 5.27 & 3.24 \\
Fe4 & 6.27 & -4.05 & -4.04 & 3.24 & 5.27 \\
\hline Singlet-II & & & & \\
\hline Fe1 & 6.25 & 5.32 & -4.03 & 3.30 & -4.18 \\
Fe2 & 6.26 & -4.03 & 5.32 & -4.18 & 3.30 \\
Fe3 & 6.26 & 3.30 & -4.18 & 5.32 & -4.03 \\
Fe4 & 6.25 & -4.18 & 3.30 & -4.03 & 5.32 \\
\hline Singlet-III & & & & \\
\hline Fe1 & 6.25 & 5.32 & -4.06 & -4.18 & 3.31 \\
Fe2 & 6.25 & -4.06 & 5.33 & 3.32 & -4.17 \\
Fe3 & 6.25 & -4.18 & 3.32 & 5.33 & -4.06 \\
Fe4 & 6.25 & 3.31 & -4.17 & -4.06 & 5.32 \\
\hline Triplet & & & & & \\
\hline Fe1 & 6.26 & 5.29 & 3.22 & -3.77 & -3.77 \\
Fe2 & 6.26 & 3.22 & 5.27 & -3.95 & -3.95 \\
Fe3 & 6.26 & -3.77 & -3.95 & 5.28 & 3.26 \\
Fe4 & 6.26 & -3.77 & -3.95 & 3.26 & 5.28 \\
\hline \hline
\end{tabular}

